\title{
Spaces of Polygonal Triangulations and Monsky Polynomials
}

\author{
Aaron Abrams · James Pommersheim
}

The first author dedicates this work to Sherman K. Stein, from whom he first learned about Monsky's Theorem.

Received: 19 March 2012 / Revised: 24 September 2013 / Accepted: 30 September 2013 /

Published online: 20 November 2013

(C) Springer Science+Business Media New York 2013

\begin{abstract}
Given a combinatorial triangulation of an $n$-gon, we study (a) the space of all possible drawings in the plane such the edges are straight line segments and the boundary has a fixed shape, and (b) the algebraic variety of possibilities for the areas of the triangles in such drawings. We define a generalized notion of triangulation, and we show that the areas of the triangles in a generalized triangulation $\mathcal{T}$ of a square must satisfy a single irreducible homogeneous polynomial relation $p(\mathcal{T})$ depending only on the combinatorics of $\mathcal{T}$. The invariant $p(\mathcal{T})$ is called the Monsky polynomial; it captures algebraic, geometric, and combinatorial information about $\mathcal{T}$. We give an algorithm that computes a lower bound on the degree of $p(\mathcal{T})$, and we present several examples in which the algorithm is used to compute the degree.
\end{abstract}

Keywords Triangulation · Area relation · Equidissection

\section{Introduction}

\subsection{Background}

This paper grew out of a desire to better understand a theorem of Paul Monsky about plane geometry. Monsky's theorem from 1970 ([11], see also [20]) states that it is impossible to cut a square into an odd number of triangles, all of the same area. This result in turn grew out of a problem that was first posed in the American Mathematical Monthly by Richman and Thomas [14] and later advanced by Thomas [21].

\footnotetext{
A. Abrams (凶)

Mathematics Department, Washington and Lee University, Lexington, VA 24450, USA

e-mail: abramsa@wlu.edu

J. Pommersheim

Department of Mathematics, Reed College, 3203 SE Woodstock Blvd, Portland, OR 97202, USA

e-mail: jamie@reed.edu 
Fig. 1 In any drawing of this

2-complex, as long as the

boundary is a parallelogram the areas will satisfy

$A-B+C-D=0$, regardless

of the placement of the central

vertex

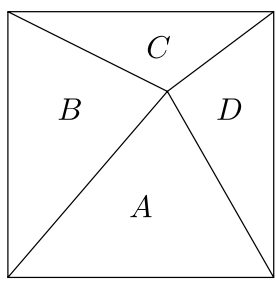

Monsky's proof blends combinatorics, topology, and number theory in an elementary but delicate way.

Monsky's result has generally been viewed as a statement about "equidissections," or tilings by equal area tiles. As such it has been generalized in various directions, e.g., to polygons other than a square $[8,12,19]$ and to higher dimensional cubes [10]. These results all have a combinatorial feel to them, and the rigidity of the notion of an equidissection is crucial.

With the hope of understanding Monsky's theorem in a different way, Joe Buhler suggested studying, for a fixed combinatorial triangulation of a square, the space of all realizable collections of areas of triangles.

Question 1 Given the combinatorics of a triangulation $\mathcal{T}$ of the unit square, which collections of areas can be realized by the triangles?

A complete answer to this question would, of course, include Monsky's theorem. One observes that the areas of the triangles add up to the area of the square, and by a dimension count, one expects the areas of the triangles to satisfy exactly one additional polynomial equation. In his senior thesis, Buhler's student Adam Robins calculated this polynomial $p(\mathcal{T})$ for several particular triangulations [17].

Example 1 If $\mathcal{T}$ is the triangulation whose combinatorial type is drawn in Fig. 1, then no matter where the central vertex is placed, the four areas must satisfy $p(\mathcal{T})=0$, where $p(\mathcal{T}):=A-B+C-D$.

A few years after [17], in an REU supervised by Serge Tabachnikov and Misha Guysinsky, students Josh Kantor and Max Maydanskiy obtained further results about this polynomial, including the calculation of several more examples and proofs of some general bounds on its degree [7]. These authors also give a family of examples showing that the degree can be exponential in the size of the triangulation.

Neither [17] nor [7] gives a rigorous proof of existence of the polynomial they study. Monsky's original theorem from [11] does prove that the areas satisfy a polynomial relation, although his approach was quite different from ours and it was initially unclear to us whether his polynomial was the same as ours. In recent communication with Monsky, however, it has become clear that his and ours are versions of the same thing. Thus in Sect. 5 we name the polynomial the Monsky polynomial.

Our approach to Monsky's polynomial is the following. We study (a) the space of all possible drawings of a given abstract triangulation $\mathcal{T}$ of an $n$-gon in which the 
boundary has a fixed shape, and (b) the variety $V(\mathcal{T})$ of possibilities for the areas of the triangles in such drawings. We establish a framework which allows the possibility of degenerating triangles by requiring that certain subsets of the vertices be collinear. For convenience we work over the complex numbers; in this framework, we show that $V(\mathcal{T})$ is an irreducible algebraic variety, and we compute its dimension. In the case $n=4, V(\mathcal{T})$ is a projective hypersurface, and so there is a single irreducible polynomial relation $p(\mathcal{T})$ that is satisfied by the areas of the triangles. This is the Monsky polynomial.

\subsection{Results}

We now give an informal summary of the results of this paper. We begin with a combinatorial triangulation $T$ of an $n$-gon. We define a triangulation $\mathcal{T}$ to be a pair $(T, C)$, where $C$ is the set of vertices of a contiguous subset of triangles of $T$. (See Fig. 2 for an example, and Sect. 2 for the precise definitions.) Intuitively, we think of $C$ as a "collinearity condition," i.e., a set of vertices which will be required to be collinear. A drawing of $\mathcal{T}$ is a placement of the vertices of $T$ in the plane with the condition that the vertices of $C$ must be collinear. We then think of the edges of the triangulation being drawn as straight line segments. We consider the space $X(\mathcal{T})$ of drawings of $\mathcal{T}$, and in Theorem 2 we show that this space is an irreducible algebraic variety.

We next consider the space of drawings of $\mathcal{T}$ in which the boundary is constrained to be a fixed shape. More precisely, if $\square$ is an $n$-gon in the plane, we define $X_{\diamond}(\mathcal{T})$ to be the subset of $X(\mathcal{T})$ consisting of those drawings in which the boundary of $T$ is mapped to an affine image of $\square$. In Theorem 3, we show that $X_{\diamond}(\mathcal{T})$ is also an irreducible algebraic variety.

Next, we introduce the area map $f_{\triangle}(\mathcal{T})$ corresponding to a triangulation $\mathcal{T}$ and a boundary shape $\square$. To do this, we let $Y(\mathcal{T})$ be the projective space with one coordinate for each non-degenerate triangle of $\mathcal{T}$. Then the rational map

$$
f_{\triangle}(\mathcal{T}): X_{\triangleright}(\mathcal{T}) \rightarrow Y(\mathcal{T})
$$

records the areas of the triangles at each point of $X_{\triangle}(\mathcal{T})$. The closure of the image of $f_{\triangle}(\mathcal{T})$ is the variety $V_{\triangle}(\mathcal{T})$ of possible areas. In Theorem 5, we show that under suitable hypotheses, $V_{\triangleright}(\mathcal{T})$ has codimension $n-3$ in $Y(\mathcal{T})$.
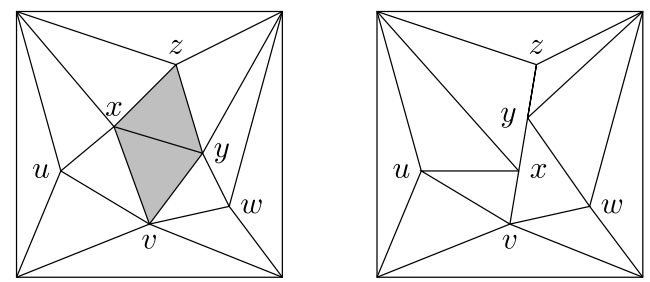

Fig. 2 On the left is a depiction of an abstract triangulation $\mathcal{T}=(T, C)$. The shading indicates the collinearity condition $C=\{v, x, y, z\}$, which is the set of vertices of the shaded triangles. On the right is (the image of) a typical drawing of the triangulation $(T, C)$. The shaded triangles have degenerated to segments because the vertices of $C$ are collinear 

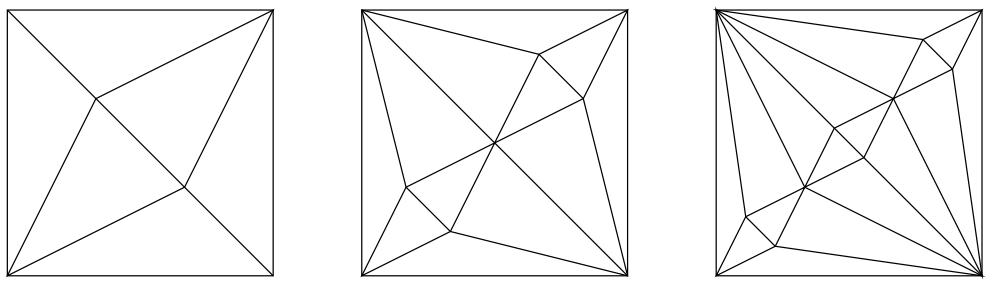

Fig. 3 The degree of $p(\mathcal{T})$ for the $k$ th triangulation in this sequence is exponential in $k$. Conjecturally, the degree is exactly $(3 k-2) 2^{k-1}$ for $k>1$. See Sect. 8

When $n=4$, so that $T$ is a quadrilateral, Theorem 5 says that $V_{\square}(\mathcal{T})$ has codimension 1 in $Y(\mathcal{T})$. Of special interest is the case that the boundary shape $\square$ is a square. In this case, we define $p(\mathcal{T})$ to be the irreducible polynomial that vanishes on $V_{\triangle}(\mathcal{T})$. The polynomial $p(\mathcal{T})$ is the Monsky polynomial.

The framework we establish allows us to successively degenerate triangles to produce new triangulations. In Sect. 6, we use this feature to give a purely combinatorial algorithm that recursively computes a lower bound for $\operatorname{deg}(\mathcal{T}):=\operatorname{deg}(p(\mathcal{T}))$. The algorithm exploits the interplay between the combinatorics of $\mathcal{T}$, the geometry of $V(\mathcal{T})$, and the algebra of $p(\mathcal{T})$.

As consequences of the algorithm, we characterize those triangulations $\mathcal{T}$ for which the degree of $p(\mathcal{T})$ is 1 , and we prove that if there is no collinearity condition $(C=\emptyset)$ and $T$ cannot be obtained by subdividing a simpler triangulation, then the degree of $p(\mathcal{T})$ is bounded below by the number of interior vertices of $T$. Then, in Sect. 8, we give an infinite family of examples for which our algorithm computes the correct degree of $\mathcal{T}$, as well as an infinite family for which the degree grows exponentially in the number of vertices of $T$. See Fig. 3.

\subsection{Further Remarks}

It is worth mentioning that for any particular $\mathcal{T}$, the polynomial $p(\mathcal{T})$ is theoretically computable: to do so is an instance of the well-known implicitization problem in algebraic geometry, whose solution involves techniques of Gröbner bases or resultants (see $[5,9])$. Unfortunately, using these general techniques to determine $p(\mathcal{T})$ is intractable in all but the smallest cases. Even when feasible, these computations give little insight into the general structure of the polynomial $p(\mathcal{T})$. In contrast, using code written by Andreae [1] for his senior thesis, we were able to run Algorithm 16 on hundreds of triangulations with a dozen or more vertices.

Our work has a similar flavor to recent work on Robbins' conjectures (see [3, 6, $13,15,16,22])$, in which a polynomial relation among squared lengths of the sides of a cyclic polygon is studied (a special case is Heron's formula). There also appear to be similarities with the well-known Bellows Conjecture (see $[2,4,18]$ ).

\section{Triangulations of Polygons}

Consider a finite 2-dimensional simplicial complex $T$ whose underlying space $|T|$ is homeomorphic to a disk. Let $\operatorname{Vertices}(T)$ denote the set of vertices of $T$. We orient 

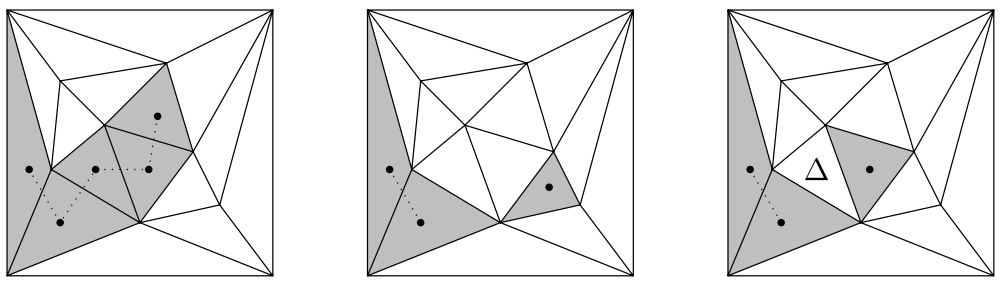

Fig. 4 In each of these figures, let $S$ denote the set of shaded triangles. The dual graph $S^{*}$ is also shown with dotted lines. On the left, $S$ is contiguous and Vertices $(S)$ is a condition. In the middle, $S$ is not contiguous and Vertices $(S)$ is not a condition. On the right, $S$ is not contiguous but Vertices $(S)$ is a condition because $S^{\prime}=S \cup \Delta$ is contiguous and $\operatorname{Vertices}\left(S^{\prime}\right)=\operatorname{Vertices}(S)$. Note $S^{\prime}=\operatorname{Triangles}(\operatorname{Vertices}(S))$

the complex and label the boundary vertices $P_{1}, \ldots, P_{n}$ in cyclic order according to the orientation. The $P_{i}$ are called the corners of $T$. The set of corners is denoted by $\partial T$. Vertices of $T$ that are not corners are called interior vertices.

It is an easy exercise to show that the number of triangles in $T$ is $2 k+n-2$, where $k$ is the number of interior vertices. If $S$ is a set of triangles of $T$ then we define a graph $S^{*}$ with one vertex for each triangle in $S$ and an edge connecting pairs of adjacent triangles of $S$. (Note that $S^{*}$ is just the subgraph of the dual graph to the triangulation $T$ spanned by the vertices that correspond to $S$.) We say that $S$ is contiguous if $S^{*}$ is a connected graph. (Equivalently, $S$ is contiguous if the interior of the union of the (closed) triangles in $S$ is connected.) We denote by $\operatorname{Vertices}(S)$ the set of vertices $v \in \operatorname{Vertices}(T)$ such that $v$ is a vertex of a triangle of $S$.

Definition 1 (Condition) A condition $C$ is a collection of vertices of $T$ such that

- $C=\operatorname{Vertices}(S)$ for some contiguous set of triangles $S$, and

- $|C \cap \partial T| \leq 2$.

See Fig. 4 for examples. We think of $C$ as a collection of vertices that will be required to be collinear; $C$ arises from "killing" a connected region of triangles.

For a given condition $C$, note that there may be more than one $S$ such that $C=$ Vertices $(S)$, but there is a uniquely determined maximal such $S$ consisting of all those triangles whose vertices are all in $C$. We denote this set of triangles by Triangles $(C)$. Again see Fig. 4.

If $C$ is a condition then we abbreviate (Triangles $(C))^{*}$ by $C^{*}$.

A triangulation $\mathcal{T}$ is a pair $(T, C)$ where $T$ is a simplicial complex as described above, and $C$ is a (possibly empty) condition.

\section{Drawings of Triangulations}

We are interested in drawing triangulations $(T, C)$ in the affine plane. We will study all drawings of $(T, C)$, first without restricting the boundary and then later with the constraint that the boundary forms a fixed shape, defined up to affine equivalence.

We will work over the field $\mathbf{C}$ of complex numbers, but many of the arguments are more easily visualized over the reals. 
A fundamental object associated to a triangulation $\mathcal{T}=(T, C)$ is the domain

$$
X=X(\mathcal{T})
$$

defined to be the set of those maps $\rho: \operatorname{Vertices}(T) \rightarrow \mathbf{C}^{2}$ such that there is an affine line $\ell \subset \mathbf{C}^{2}$ containing $\rho(v)$ for every vertex $v$ of the condition $C$. Note that $X(\mathcal{T})$ is an affine subvariety of the space of all maps $\operatorname{Vertices}(T) \rightarrow \mathbf{C}^{2}$. Each point $\rho \in X(\mathcal{T})$ is called a drawing of $\mathcal{T}$.

Theorem 2 If $\mathcal{T}$ is a triangulation, then $X(\mathcal{T})$ is an irreducible algebraic variety over $\mathbf{C}$.

Proof Denote the interior vertices of $T$ by $v_{1}, \ldots, v_{k}$. Consider any total order < on Vertices $(T)$ satisfying $P_{i}<v_{j}$ for all $i$ and $j$. For $v \in \operatorname{Vertices}(T)$ let $\alpha(v)=1$ if $v \in C$ and there are at least two vertices $x_{1}, x_{2} \in C$ with $x_{i}<v$. Otherwise let $\alpha(v)=2$. Then, if we draw $\mathcal{T}$ by placing the vertices in order, then $\alpha(v)$ is the number of degrees of freedom we have in placing $v$.

We parameterize $X$ by defining a rational map

$$
g: \prod_{v \in \operatorname{Vertices}(T)} \mathbf{C}^{\alpha(v)} \longrightarrow X .
$$

Let $x=\left(x_{v}\right)_{v \in \operatorname{Vertices}(T)}$ be coordinates on the domain $\prod_{v \in \operatorname{Vertices}(T)} \mathbf{C}^{\alpha_{v}}$. The coordinate functions $\rho(v)(x)$ of the point $g(x)$ are constructed inductively, as follows. For each $v \in \operatorname{Vertices}(T)$, we assume that the coordinate functions $\rho(w)(x)$ for vertices $w$ with $w<v$ have been defined. Then, if $\alpha(v)=2$, we set $\rho(v)(x)=x_{v}$. If $\alpha(v)=1$, then set $\rho(v)(x)=x_{v} \rho(y)(x)+\left(1-x_{v}\right) \rho(z)(x)$ where $y$ and $z$ are the first two points of $C$ and $y<z$.

Clearly, the image of $g$ is contained in $X$. It then follows that $X$ is irreducible, provided that the image of $g$ is a Zariski dense subset of $X$. To show this, take any drawing $\rho \in X$. Note that $\rho$ is in the image of $g$ iff either $\rho(y) \neq \rho(z)$ or all the points $\rho(v)$ coincide for $v \in C$. Hence if $\rho$ is not in the image of $g$, then $\rho(y)=\rho(z)$ but the line $\ell$ is uniquely defined. By perturbing $\rho(z)$ along $\ell$, we obtain a nearby point $\rho^{\prime}$ of $X$ that is in the image of $g$. Thus the image of $g$ is dense in $X$, and $X$ is irreducible.

The next result is similar, but we impose a boundary condition. Given $n \geq 3$, an $n$-gon shape $\triangle$ is an ordered $n$-tuple $\left(z_{1}, \ldots, z_{n}\right)$ of points in $\mathbf{C}^{2}$ such that no three are collinear. Suppose $\mathcal{T}=(T, C)$ is given with $|\partial T|=n$, and let $\square$ be an $n$-gon shape. We let $X_{\triangleright}(\mathcal{T})$ be the set of drawings of $\mathcal{T}$ whose boundary is an affine image of $\square$; that is,

$$
X_{\triangle}(\mathcal{T})=\left\{\rho \in X(\mathcal{T}) \mid \exists \phi \in \widehat{\operatorname{Aff}} \text { with } \rho\left(P_{i}\right)=\phi\left(z_{i}\right) \forall i\right\},
$$

where $\widehat{\text { Aff }}$ is the set of (not necessarily invertible) affine transformations of $\mathbf{C}^{2}$.

Theorem 3 If $\mathcal{T}$ is a triangulation with $n$ boundary vertices and $\triangle$ is an $n$-gon shape, then $X_{\square}(\mathcal{T})$ is an irreducible algebraic variety over $\mathbf{C}$. 
Proof We proceed basically as in the previous theorem, with some modifications. We order the vertices in any way as long as $P_{i}<v_{j}$ for all $i$ and $j$ and the vertices of $C \cap \partial T$ come first. Set $\alpha(v)=2$ for the first three vertices in the order, set $\alpha(v)=0$ for any remaining boundary vertices, and define $\alpha\left(v_{i}\right)$ as before.

We parameterize $X_{\triangle}$ again by $\prod_{v \in \operatorname{Vertices}(T)} \mathbf{C}^{\alpha(v)}$. Drawing the vertices in order, we see that the first three points determine the rest of the boundary as well as the affine map $\phi$. For the $v_{i}$, the parameterization works as in Theorem 2.

Again we wish to show that the image of the parameterization is dense in $X_{\square}(\mathcal{T})$. Points $\rho \in X_{\triangle}(\mathcal{T})$ in the image of the parameterization are characterized exactly as before, and if a point $\rho$ of $X$ is not in the image then by our choice of ordering on vertices, the second point of the condition has $\alpha=2$, and so we can move it along $\ell$ as in the previous proof.

Remarks The above theorems easily generalize to triangulations with several collinearity conditions, as long as the conditions are disjoint. The hypotheses on the condition $C$ are also unnecessary for irreducibility; one could allow $C$ to be an arbitrary subset of $\operatorname{Vertices}(T)$.

With some additional arguments, the above theorems also generalize to the socalled dissections, which is the context in which Monsky made his original discoveries. A dissection is a decomposition of the polygon into closed triangles whose interiors are disjoint; the decomposition need not be simplicial. Every dissection can be viewed as a (simplicial) triangulation with possibly many collinearity conditions.

Perhaps the most general setting of all is that of triangulations with arbitrary collinearity conditions that are allowed to intersect. One difficulty that arises in this situation is that the conditions may not be logically independent, meaning that it is possible for one to follow from the others. For example, this could be the result of an incidence theorem such as Pappus' Theorem. In such a case, $X$ may not have the expected dimension and it becomes more difficult to parameterize.

Another difficulty with this generalization is that it can cause $X$ to be reducible. This happens, for instance, if $X$ contains one condition of the form $\{w, x, y\}$ and another condition of the form $\{x, y, z\}$. In this case, $X$ will contain two components, one consisting of drawings in which $w, x, y, z$ are all drawn collinearly and one consisting of drawings in which $x$ and $y$ are drawn copointally. See Example 2.

We believe that if every pair of collinearity conditions intersects in at most one vertex, then the space of drawings is irreducible.

\section{The Variety of Areas}

If $\Delta$ is an oriented triangle in $\mathbf{C}^{2}$ with vertices $\left(x_{1}, y_{1}\right),\left(x_{2}, y_{2}\right),\left(x_{3}, y_{3}\right)$ then the area of $\Delta$ is given by the formula

$$
\operatorname{Area}(\Delta)=\frac{1}{2}\left|\begin{array}{ccc}
1 & 1 & 1 \\
x_{1} & x_{2} & x_{3} \\
y_{1} & y_{2} & y_{3}
\end{array}\right| .
$$

This is the usual (signed) area when $\Delta \subset \mathbf{R}^{2}$. 

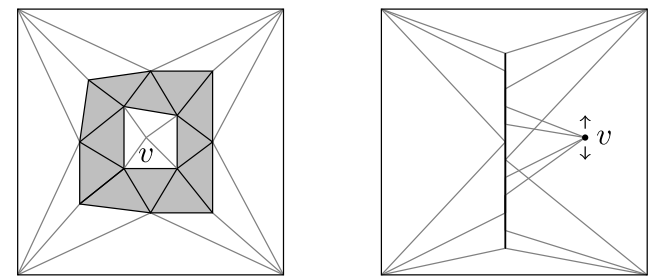

Fig. 5 If $C$ consists of the vertices of the shaded triangles, then $C$ is a condition that isn't non-separating. In any drawing of $T$, there is a line containing all the vertices of $C$, and the vertex $v$ can move parallel to this line without changing the area of any triangle

Let the triangulation $\mathcal{T}$ be given, let $n$ be the number of boundary points, and let $\checkmark$ be a fixed $n$-gon shape. Let $Y=Y(\mathcal{T})$ be the projective space with a coordinate $A_{i}$ for each triangle $\Delta_{i}$ whose vertices are not all in $C$. We have rational maps $f=$ $f(\mathcal{T}): X(\mathcal{T}) \rightarrow Y(\mathcal{T})$ and $f_{\triangle}(\mathcal{T}): X_{\triangle}(\mathcal{T}) \rightarrow Y(\mathcal{T})$ called the area maps given by

$$
f_{*}(\rho)=\left[\cdots: \operatorname{Area}\left(\rho\left(\Delta_{i}\right)\right): \cdots\right] .
$$

The main object of study in this paper is the closure of the image of $f_{\triangle}$, denoted by $V_{\square}=V_{\square}(\mathcal{T})$. This is an irreducible (projective) subvariety of $Y$.

The goal of this section is to establish the dimension of $V_{\bullet}$. For a point $\rho$ of $X_{\triangle}$ with $\rho\left(P_{1}\right), \rho\left(P_{2}\right), \rho\left(P_{3}\right)$ not collinear, the orbit of $\rho$ under the action of the group Aff of invertible affine transformations of $\mathbf{C}^{2}$ is contained in a single fiber of $f_{\triangle}$. Thus generic fibers have dimension at least 6 (the dimension of Aff).

Exactly two phenomena can cause fibers to be larger than this. First, consider the subgraph $G$ of (the 1-skeleton of) $T$ spanned by those vertices that are not in $C$. If this graph has a connected component that contains none of the $P_{i}$, then this component can be "sheared" with respect to $\ell$, independently of the rest of $G$, without changing the area of any triangle. Thus there is at least one additional 1-parameter family of drawings inside the fiber for each such component. See Fig. 5.

Definition 4 (Non-separating) Let $\mathcal{T}=(T, C)$. Let $G$ be the graph consisting of those edges of $T$ whose both endpoints are not in $C$. The condition $C$ is nonseparating if every connected component of $G$ contains at least one corner of $T$.

(Recall that a corner is a vertex of $\partial T$.) Note that it is possible to have $C$ nonseparating and $G$ disconnected by having $C$ contain two non-adjacent corners.

Second, we call a vertex $v \in C$ a mosquito if all neighbors of $v$ are also in $C$. (In particular, $v$ is not in $\partial T$.) If $v$ is a mosquito then we may form a new triangulation $\mathcal{T}^{\prime}=\left(T^{\prime}, C^{\prime}\right)$ as follows: $T^{\prime}$ is obtained from $T$ by deleting $v$ and re-triangulating the star of $v$ with no interior vertices, and $C^{\prime}$ is obtained from $C$ by deleting $v$. Let $f_{\triangle}^{\prime}$ be the corresponding area map for $\mathcal{T}^{\prime}$, and note that the image of $f_{\triangle}^{\prime}$ is exactly equal to the image of $f_{\triangle}$. Thus the presence of $v$ in $T$ gives rise to an independent dimension in the fiber of $f_{\triangle}$. See Fig. 6. 
Fig. 6 The vertex $m$ is a mosquito in the $(T, C)$ shown on the left
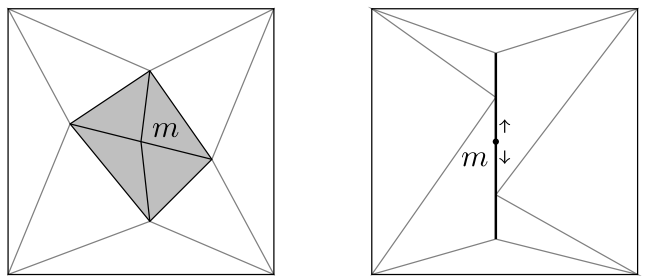

Fig. 7 If $S$ is the set of shaded triangles, then the condition $C=\operatorname{Vertices}(S)$ is not non-separating. Note that $S$ is contiguous but Triangles $(C)$ includes the triangle vxy and is not contiguous

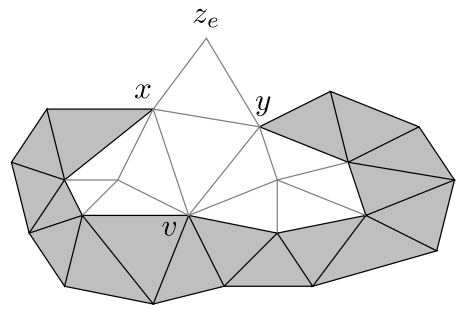

Theorem 5 Let $\mathcal{T}=(T, C)$ be a triangulation with $|\partial T|=n$, and let $\square$ be an $n$ gon shape. Assume $C$ is non-separating, and let $m$ be the number of mosquitos in $\mathcal{T}$. Then:

(a) the dimension of $V_{\triangle}(\mathcal{T})$ is $\operatorname{dim} X_{\square}-m-6$;

(b) the codimension of $V_{\triangle}(\mathcal{T})$ in $Y$ is $n-3$.

We now prove some preliminary lemmas.

Lemma 6 Let $\mathcal{T}=(T, C)$ be a triangulation. If $C$ is non-separating then Triangles $(C)$ is contiguous, i.e., the graph $C^{*}$ is connected. If in addition $\mathcal{T}$ has no mosquitos then $C^{*}$ is a tree.

Proof Assume $C$ is non-separating, and suppose $C^{*}$ is not connected. Let $S$ be a contiguous set of triangles such that $C=\operatorname{Vertices}(S)$. The union of the triangles of Triangles $(C)$ is a connected subset of $T$. Since $S$ is contiguous, $S^{*}$ is connected and therefore contained in a single component of $C^{*}$, called the main component.

Let $A$ be the union of the triangles making up a component of $C^{*}$ other than the main component. For each edge $e$ of $\partial A$ that is not in $\partial T$, there is a vertex $z_{e}$ of $T$ such that $\left\{z_{e}\right\} \cup \partial e$ is a triangle of $T \backslash A$. See Fig. 7. Note that $z_{e}$ is not in $C$. Let $e=x y$ be one such edge. Note both $x$ and $y$ are in $C$, and neither of the triangles containing the edge $x y$ is in $S$. Thus there is an arc from $x$ to $y$ in $S$, and this arc together with the edge $x y$ forms a loop $\gamma$ in $T$. This loop encloses either $z_{e}$ or all the $z_{f}$ for $f \neq e$. In either case, the hypothesis that $C$ is non-separating is contradicted. We conclude that $C^{*}$ is connected.

Finally, observe that any loop in $C^{*}$ would enclose some vertices of $T$. If all such vertices are in $C$ they would be mosquitos, but if some vertex is not in $C$ then $C$ would fail to be non-separating. Thus if $\mathcal{T}$ has no mosquitos then $C^{*}$ is a tree. 
Fig. 8 A new triangle $x y z$ could not already have $z \in C$

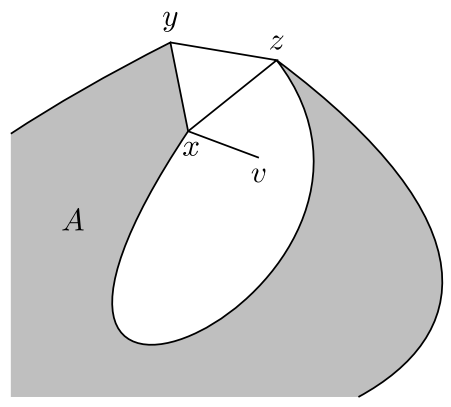

Lemma 7 Let $\mathcal{T}=(T, C)$ be a triangulation with no mosquitos and with $C$ nonempty and non-separating. Then $\mid$ Triangles $(C)|=| C \mid-2$.

Proof Note that Triangles $(C)$ consists of a single triangle if and only if $|C|=3$.

By the previous lemma, the graph $C^{*}$ is a tree. We think of building the tree $C^{*}$ by starting with a vertex and adding leaves. Suppose at some step we have a collection of triangles $A$, and we wish to add a new triangle $x y z$. The new triangle contributes 1 to $\mid$ Triangles $(C) \mid$. The new triangle contributes at most 1 to $|C|$, because the triangle $x y z$ shares an edge, say $x y$, with $A$. We now show that $z$ was not already in $A$, and hence the triangle $x y z$ contributes exactly 1 to $|C|$.

The argument is similar to the argument that $C^{*}$ contains no cycles; see Fig. 8. Suppose on the contrary that $z$ is a vertex of a triangle of $A$. Let $w$ be the midpoint of the edge $x y$. Then $z$ is connected to $w$ by an arc in $A$. This arc together with the line segment from $w$ to $z$ in the triangle $x y z$ makes a loop $\gamma$ that separates $x$ from $y$. Relabel if necessary so that $x$ is in the bounded region determined by $\gamma$. Now, since $\mathcal{T}$ has no mosquitos, $x$ must have a neighbor $v$ that is not in $C$. But $v$ is also in the bounded region determined by $\gamma$, contradicting the hypothesis that $C$ is non-separating.

The proof of Theorem 5 is an induction based on contracting an edge of $T$. In order to define a contraction of a triangulation $\mathcal{T}=(T, C)$, we need some language and notation.

First, we say that $T$ contains a subdivision if there are vertices $x, y, z$ of $T$ such that $x y, y z, x z$ are edges of $T, x y z$ is not a triangle of $T$, and $\{x, y, z\} \neq \partial T$.

Next, an edge $e=x y$ of $T$ is contractible if $e \not \subset \partial T$ and the "contraction" $T / e$ (defined below) is a simplicial complex. Specifically, this means that there are exactly two vertices $w, z$ of $T$ that are adjacent to both $x$ and $y$; when this holds, we define $T / e$ to be the simplicial complex obtained from $T$ by deleting the triangles $w x y$ and $x y z$ and identifying the vertices $x$ and $y$ (and consequently identifying the edges $w x$ and $w y$, and the edges $x z$ and $y z$ ).

We leave it to the reader to verify that if the above condition holds, then $T / e$ is indeed simplicial. See Fig. 9.

Note in the case that $e=x y$ is not a contractible edge, either $e$ is on the boundary or $T$ contains a subdivision. Said differently, if $T$ has no subdivisions, then every non-boundary edge of $T$ is contractible. 
Fig. 9 The figure on the left shows a contractible edge $e$, as long as there is no vertex $v$ as there is in the right figure. The edge $e$ on the right is not contractible, regardless of how the interior of the quadrilateral $x w y v$ is triangulated
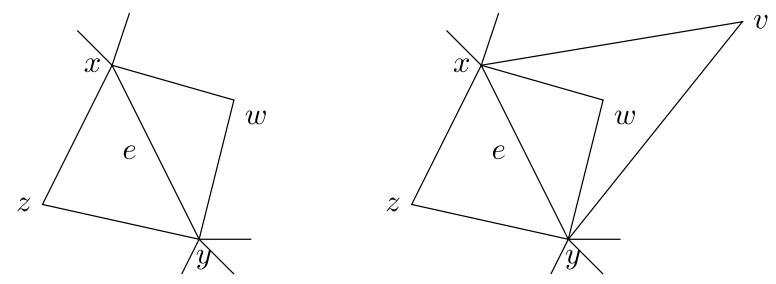

Fig. 10 If $C$ consists of the vertices of the four triangles shown on the left, then even if the edge $e$ is contractible, it is not good, because $C / e$ is not a condition

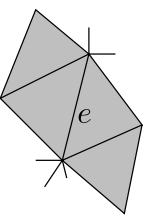

C

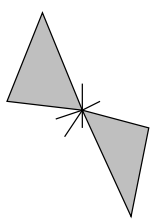

$C / e$

Suppose $\mathcal{T}=(T, C)$ is a triangulation and $e=x y$ is a contractible edge of $T$. If $w x y$ and $x y z$ are triangles of $T$ (with $w \neq z$ ) then we define $C / e=$ Vertices(Triangles $(C) \backslash\{w x y, x y z\}) \subset \operatorname{Vertices}(T / e)$. Thus $C / e=\emptyset$ if $C \subset\{w, x$, $y, z\}$, and otherwise $C / e$ is the image of $C$ under the quotient map $\operatorname{Vertices}(T) \rightarrow \operatorname{Vertices}(T / e)$. Note, however, that $C / e$ may not be a condition since contracting $e$ may destroy contiguity. See Fig. 10.

To ensure that $C / e$ is a condition, we introduce one more concept.

Definition 8 (Good edge) Suppose that $\mathcal{T}=(T, C)$ is a triangulation with $C$ nonseparating. An edge $e=x y$ of $T$ is $\operatorname{good}$ if $e$ is contractible and either

Type $1\{x, y\} \cap C=\emptyset$, or

Type $2 e \subset \partial C$, i.e., $e \subset C$ and there exist $w, z \in \operatorname{Vertices}(T)$ adjacent to both $x$ and $y$, with $w \in C, z \notin C$.

Remark Note that if $T$ has at least one interior vertex and no subdivisions, then $T$ has good edges. The following lemma allows us to contract these edges.

Lemma 9 Let $\mathcal{T}=(T, C)$ be a triangulation with $C$ non-separating. If e is a good edge then $C / e \subset \operatorname{Vertices}(T / e)$ is a non-separating condition in the triangulation $T / e$.

Proof Let $S$ be a contiguous set of triangles of $T$ such that $C=\operatorname{Vertices}(S)$.

If $e$ is a type 1 good edge, then $C / e$ consists of the same vertices as $C$, and $S$ remains contiguous in $T / e$. Thus $C / e$ is a condition. Also, the complement of $C / e$ in $T / e$ is just a contraction of the complement of $C$ in $T$. Since $C$ is non-separating, so is $C / e$.

Now suppose $e$ is a type 2 good edge. Let $S / e$ be the image of $S$ under the contraction. Then $S / e$ is contiguous, and $C / e=\operatorname{Vertices}(S / e)$. So $C / e$ is a condition. 
Also, the complement of $C / e$ in $T / e$ is identical to the complement of $C$ in $T$, so $C / e$ is non-separating.

Thus the good edges are the ones we will contract: if $\mathcal{T}=(T, C)$ and $e$ are as in the lemma, we define $\mathcal{T} / e$ to be the triangulation $(T / e, C / e)$. We also sometimes denote this by $\left.\mathcal{T}\right|_{x=y}$.

We are now ready to prove Theorem 5 .

Proof of Theorem 5 First, we show that it is possible to eliminate mosquitos. Suppose that $v \in \operatorname{Vertices}(T)$ is a mosquito. Let $<$ be the total order on Vertices $(T)$ used in the proof of Theorem 3, and let $\alpha$ be defined as it was in that proof. Without loss of generality, we can assume that $v$ is the last vertex with respect to the chosen ordering. It follows that $\alpha(v)=1$. As we mentioned in the discussion preceding the theorem, we may form a new triangulation by eliminating $v$ and retriangulating the star of $v$. This reduces the dimension of $X_{\triangleright}$ by $\alpha(v)=1$, reduces $m$ by 1 , and leaves $Y$ and the image of $f$ unchanged. Thus, by induction, we may assume that $m=0$.

We claim that for any $\mathcal{T}$ satisfying the hypotheses, conclusions (a) and (b) are equivalent. Because $m=0$, this follows from the equation $\operatorname{dim} Y=\operatorname{dim} X+n-9$, which we now verify. Suppose that $C=\emptyset$. Then $\operatorname{dim} X=\sum \alpha(v)=6+2 k$. Also, recall that the number of triangles of $T$ is $2 k+n-2$, so $\operatorname{dim} Y=2 k+n-3$, as desired. On the other hand, if $C \neq \emptyset$, then $\operatorname{dim} X=\sum \alpha(v)=6+2 k-(|C|-2)$. Also, $\operatorname{dim} Y=2 k+n-3-\mid$ Triangles $(C) \mid$. The claim now follows from Lemma 7.

We now prove (a) and (b) together by induction on the number $k$ of interior vertices. If $k=0$, then $C=\emptyset$ and $m=0$, so $\operatorname{dim} X_{\square}=6, \operatorname{dim} Y=n-3$, and $f$ is a constant map. So (a) and (b) hold.

Suppose that $\mathcal{T}$ contains a subdivision with vertices $x, y, z$. We may assume that not all of $x, y, z$ are in $C$, by the hypothesis that $C$ is non-separating. In this case, we can form new triangulations $\mathcal{T}^{\prime}$ and $\mathcal{T}^{\prime \prime}$ as follows. We get $T^{\prime}$ by deleting all vertices (and triangles) in the interior of the triangle $x y z$ and replacing them with the single triangle $x y z$; note that $\partial T^{\prime}$ is an $n$-gon. We let $C^{\prime}=\operatorname{Vertices}(\operatorname{Triangles}(C) \cap$ Triangles $\left.\left(T^{\prime}\right)\right)$ and $\mathcal{T}^{\prime}=\left(T^{\prime}, C^{\prime}\right)$.

We get $T^{\prime \prime}$ by taking the sub-triangulation of $T$ consisting of those triangles inside $x y z$. The boundary of $T^{\prime \prime}$ is a 3-gon. Let $C^{\prime \prime}=\operatorname{Vertices}(\operatorname{Triangles}(C) \cap$ Triangles $\left.\left(T^{\prime \prime}\right)\right)$ and $\mathcal{T}^{\prime \prime}=\left(T^{\prime \prime}, C^{\prime \prime}\right)$.

There is a projection $\hat{\pi}$ from $Y$ to $Y^{\prime}$ that replaces the coordinates corresponding to triangles of $T^{\prime \prime}$ with their sum. Note that $\hat{\pi}$ restricts to a projection $\pi: V \rightarrow V^{\prime}$. Let $v^{\prime}$ be a generic point of $V^{\prime}$, i.e., $v^{\prime} \in \operatorname{Im}\left(f^{\prime}\right)$ and the coordinate corresponding to the sum of triangles from $T^{\prime \prime}$ is nonzero. (We can do this because this coordinate is not always zero, which follows from the assumptions that $m=0$ and $C$ is nonseparating.) We claim that $\pi^{-1}\left(v^{\prime}\right)=\hat{\pi}^{-1}\left(v^{\prime}\right)$. Clearly, $\pi^{-1}\left(v^{\prime}\right) \subseteq \hat{\pi}^{-1}\left(v^{\prime}\right)$. Let $v \in$ $\hat{\pi}^{-1}\left(v^{\prime}\right)$; we wish to show $v \in V$.

To see this, let $\rho^{\prime} \in X^{\prime}$ with $f_{\triangle}^{\prime}\left(\rho^{\prime}\right)=v^{\prime}$. In the drawing $\rho^{\prime}$, the triangle $x y z$ has a shape $\nabla$. Consider the map $f_{\nabla}$. By induction, the codimension of its image is zero, which means there is a drawing of $\nabla$ realizing the areas $\left(v_{\Delta}\right)$ where $\Delta$ runs through the triangles of $T^{\prime \prime}$. Inserting this drawing of $\nabla$ produces a drawing $\rho \in X$ with $f_{\triangle}(\rho)=v$. 
Now that we know that $\pi^{-1}\left(v^{\prime}\right)=\hat{\pi}^{-1}\left(v^{\prime}\right)$, we conclude that $\operatorname{dim} V=\operatorname{dim} V^{\prime}+$ $\operatorname{dim} \pi^{-1}\left(v^{\prime}\right)=\operatorname{dim} V^{\prime}+\operatorname{dim} V^{\prime \prime}$. Since we also have $\operatorname{dim} Y=\operatorname{dim} Y^{\prime}+\operatorname{dim} Y^{\prime \prime}$, we get $\operatorname{codim}_{Y} V=\operatorname{codim}_{Y^{\prime}} V^{\prime}+\operatorname{codim}_{Y^{\prime \prime}} V^{\prime \prime}=\operatorname{codim}_{Y^{\prime}} V^{\prime}$. Now the induction hypothesis applied to $\mathcal{T}^{\prime}$ implies that $\operatorname{codim}_{Y} V=n-3$, proving that (b) (and hence also (a)) holds for $\mathcal{T}$.

The remaining case is that $\mathcal{T}$ contains no subdivisions. We will prove (a) (under the assumption $m=0$ ); we first note that the inequality $\operatorname{dim} V_{\square}(\mathcal{T}) \leq \operatorname{dim} X_{\square}-6$ follows from the previous discussion about the fibers of $f$.

For the opposite inequality, recall that we are in the following situation. The triangulation $\mathcal{T}$ has no mosquitos, no subdivisions, and some number $k>0$ of interior vertices. As we remarked earlier, in this setting there exists a good edge $e=x y$ of $T$. By Lemma 9, the induction hypothesis applies to the contraction $\mathcal{T} / e$, which we now denote $\mathcal{T}^{\prime}$. Furthermore, from the definition of goodness it follows that $\mathcal{T}^{\prime}$ has no mosquitos. Thus, inductively, the image of $f_{\square}^{\prime}$ has dimension at least $\operatorname{dim} X^{\prime}-6$.

Now let $w$ and $z$ be the vertices of $T$ that are adjacent to both $x$ and $y$. Choose $\rho^{\prime} \in X^{\prime}$ such that $w, x, z$ are not collinear and such that the rank of $D f^{\prime}\left(\rho^{\prime}\right)$ is maximal. This can be done because, by the definition of goodness, we may assume $w, x, z$ are not all contained in $C$. We view $X^{\prime}$ as the subset of $X$ where $\rho(y)=\rho(x)$, and $f_{\triangle}^{\prime}$ as a restriction of $f_{\triangle}$.

Suppose $e$ is a type 1 good edge. Then $\operatorname{dim} X=\operatorname{dim} X^{\prime}+2$, so our goal is to show that $\operatorname{dim} \operatorname{Im}\left(f_{\triangle}\right) \geq \operatorname{dim} \operatorname{Im}\left(f_{\triangle}^{\prime}\right)+2$. It suffices to show that the rank of $D f_{\triangle}$ at $\rho^{\prime}$ is at least two more than the rank of $D f^{\prime}$ at $\rho^{\prime}$. This is achieved by moving $y$ off $x$ in the two independent directions $\rho(w)-\rho(x)$ and $\rho(z)-\rho(x)$. These tangent directions in $X$ map via $D f_{\triangle}$ to tangent directions in $Y$ that are independent of each other and of the image of $D f^{\prime}$, because in the image of $D f^{\prime}$ the areas of both $w x y$ and $x y z$ are constantly zero, and moving in the direction $\rho(w)-\rho(x)$ keeps $w x y$ at zero but changes $x y z$ whereas moving in the direction $\rho(z)-\rho(x)$ keeps $x y z$ at zero but changes $w x y$. See Fig. 11.

On the other hand, if $e$ is a type 2 good edge, then $x, y \in C$ and (after relabeling if necessary) we may assume that $w \notin C$ and the order $<$ is such that $x<y$ and $\alpha(y)=1$. This choice of $<$ induces an ordering on the vertices of $\mathcal{T} / e$ in which the sum of the $\alpha$ 's has decreased by one. Hence, in this case we only need to show that the rank of $D f_{\triangle}$ at $\rho^{\prime}$ is at least one more than the rank of $D f^{\prime}$ at $\rho^{\prime}$. Here, this is achieved by moving $y$ off $x$ in the direction of the line $\ell$ associated to $C$. This changes the area of $w x y$ and hence is independent of all directions in the image of $D f^{\prime}$.

This establishes the inequality $\operatorname{dim} f \geq \operatorname{dim} X-m-6$ and shows that (a) (and hence (b)) holds for $\mathcal{T}$.
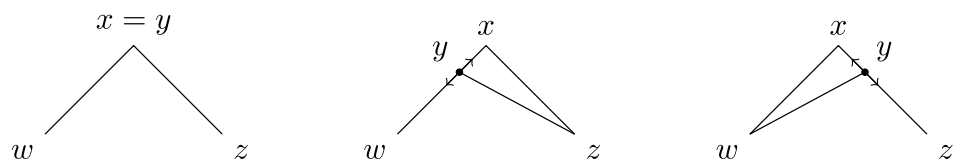

Fig. 11 The case $\alpha(y)=2$ : as $y$ moves off of $x$ in the indicated directions, it generates two new tangent directions in area space 


\section{Squares and the Monsky Polynomial}

We now turn our attention to triangulations of a square, i.e., $|\partial T|=4$. We rename the corners $P, Q, R, S$. By Theorem 5 , if $\mathcal{T}=(T, C)$ is a triangulation with $|\partial T|=4$, then for any boundary shape, the (irreducible) projective variety $V_{\triangle}$ has codimension 1 inside the projective space $Y$.

Corollary 10 Let $\mathcal{T}=(T, C)$ be a triangulation with $|\partial T|=4$. Then for any 4-gon shape $\square, V_{\triangle}(\mathcal{T})$ is the zero set of a single irreducible homogeneous polynomial.

Definition 11 (Monsky polynomial and degree) Let $\mathcal{T}=(T, C)$ be a triangulation with $|\partial T|=4$, and let $\square$ be any nondegenerate parallelogram. We define the Monsky polynomial $p(\mathcal{T})$ to be the unique irreducible polynomial that vanishes on $V(\mathcal{T}):=V_{\square}(\mathcal{T})$, scaled so the coefficients are integers with no common factor. This polynomial is well-defined up to sign. We also define the degree of $\mathcal{T}$ to be $\operatorname{deg}(\mathcal{T})=\operatorname{deg}(p(\mathcal{T}))$.

Note that the scaling of $p(\mathcal{T})$ in the above definition is possible since $V(\mathcal{T})$ is the image of the map $f:=f_{\square}(\mathcal{T})$ whose coordinate functions are polynomials with rational coefficients.

\section{A Degree Algorithm}

In this section, we study the Monsky polynomial $p(\mathcal{T})$. In particular, we describe an algorithm for computing a lower bound on the degree of $p(\mathcal{T})$. The degree $\operatorname{deg}(\mathcal{T})$ is an integer invariant of $\mathcal{T}$. At present we do not know how $\operatorname{deg}(\mathcal{T})$ is related to any classical graph-theoretic invariants of the 1-skeleton of $T$.

The idea of the algorithm is to intersect $V$ with various coordinate hyperplanes, and to consider the components of the intersection inductively. We can identify all the components of intersection, and the sum of the degrees of these components gives a lower bound for the degree of $V$. If each component occurs with multiplicity one, then this sum is exactly the degree of $V$. However, it is difficult to identify the multiplicities of the components, which is why we can prove only that our algorithm produces a lower bound on the degree. On the other hand, the algorithm involves a choice of ordering of the triangles of $T$, and in all cases we have observed, there is an ordering that produces the correct degree. We conjecture that this is always the case. See the discussion at the end of the section.

For the rest of this section, we let $\mathcal{T}=(T, C)$ be given, where $|\partial T|=4$ and $C$ is a non-separating condition. Let $p(\mathcal{T})$ be the Monsky polynomial of $\mathcal{T}$.

Our algorithm is recursive, and we will need a way to detect triangulations with $\operatorname{deg} \mathcal{T}=1$. For this purpose, we introduce two types of triangulations where it is easy to see that the equation is linear. Later, we shall see that any $\mathcal{T}$ for which $\operatorname{deg} \mathcal{T}=1$ is of one of these types.

For a triangulation $\mathcal{T}=(T, C)$, we say that $x, y \in \operatorname{Vertices}(T)$ are joined if either $\{x, y\} \subset C$ or there is an edge $x y$ in the 1 -skeleton of $T$. 
Definition 12 (Types $\mathrm{D}$ and $\mathrm{X}$ ) Let $\mathcal{T}=(T, C)$ be a triangulation. We say that $\mathcal{T}$ is linear of type $D$ if there are two opposite corners of $T$ that are joined. We say that $\mathcal{T}$ is linear of type $X$ if there is a vertex of $T$ that is joined to all four corners of $T$.

If $\mathcal{T}$ is linear of type $\mathrm{D}$ or $\mathrm{X}$, then there is an obvious linear relation among the areas, so $\operatorname{deg} \mathcal{T}=1$. (The letter D stands for "diagonal"; for an example of type X, see Fig. 1.)

The next lemma shows that we can eliminate subdivisions without affecting the degree. Suppose $T$ contains a subdivision with vertices $x, y, z$; then as in the proof of Theorem 5 we can form a new triangulation $\mathcal{T}^{\prime}=\left(T^{\prime}, C^{\prime}\right)$ as follows. We get $T^{\prime}$ by deleting all vertices (and triangles) in the interior of the triangle $x y z$ and replacing them with the single triangle $x y z$, and we set $C^{\prime}=C \cap \operatorname{Vertices}\left(T^{\prime}\right)$.

Lemma 13 Let $\mathcal{T}=(T, C)$ be a triangulation with $C$ non-separating. Suppose $T$ contains a subdivision with vertices $x, y, z$ and define $\mathcal{T}^{\prime}$ as above. Then the polynomial $p(\mathcal{T})$ is obtained from $p\left(\mathcal{T}^{\prime}\right)$ by replacing the variable $A_{x y z}$ with the linear form $\sum \Delta_{i}$ where the sum is over the triangles in $T$ interior to $x y z$. In particular, $\operatorname{deg}(\mathcal{T})=\operatorname{deg}\left(\mathcal{T}^{\prime}\right)$.

Proof As in the proof of Theorem 5, we have a projection $\hat{\pi}: Y \rightarrow Y^{\prime}$ that restricts to a projection $\pi: V \rightarrow V^{\prime}$. Note that $p\left(\mathcal{T}^{\prime}\right) \circ \hat{\pi}$ defines an irreducible homogeneous polynomial on $Y$. This polynomial vanishes on $V$ because $v \in V$ implies $\hat{\pi}(v)=$ $\pi(v) \in V^{\prime}$. Therefore, this polynomial equals $p(\mathcal{T})$.

A key step in our algorithm involves a choice of triangle to kill. The next lemma asserts that in most situations we can choose such a triangle, preserving the nonseparating property of the condition.

Definition 14 (Killable triangle) Let $\mathcal{T}=(T, C)$ be a triangulation with $C$ nonseparating. A triangle $\Delta$ of $T$ is called killable if $\Delta \notin \operatorname{Triangles}(C)$ and $C \cup$ $\operatorname{Vertices}(\Delta)$ is a non-separating condition.

Lemma 15 Fix $\mathcal{T}=(T, C)$ with $C$ non-separating and such that $T$ has no subdivisions. Then there exists a killable triangle in $\mathcal{T}$, or else $\mathcal{T}$ is linear of type $D$ or $X$.

Proof We will assume that $\mathcal{T}$ is not linear of type D or X, and we will show that there is a killable triangle.

If $C=\emptyset$ then any triangle that does not contain three corners of the square is killable. If each triangle contains three corners of the square then the triangulation has exactly two triangles and $\mathcal{T}$ is linear of type $\mathrm{D}$. Henceforth, we assume $C \neq \emptyset$.

Recall that because $T$ has no subdivisions, every edge $e$ of $\partial C$ that is not contained in $\partial T$ is good (see Definition 8). For each such edge, there is a triangle $\Delta_{e}$ containing $e$ that is adjacent to a triangle of Triangles $(C)$. We will show that at least one of these $\Delta_{e}$ 's is killable. Denote by $v_{e}$ the vertex of $\Delta_{e}$ that is not in $C$; then Vertices(Triangles $\left.(C) \cup \Delta_{e}\right)=C \cup\left\{v_{e}\right\}$. 
Fig. 12 If $C \cup v_{e}$ is separating, then there is a killable triangle in the bounded region separated by $C \cup v_{e}$

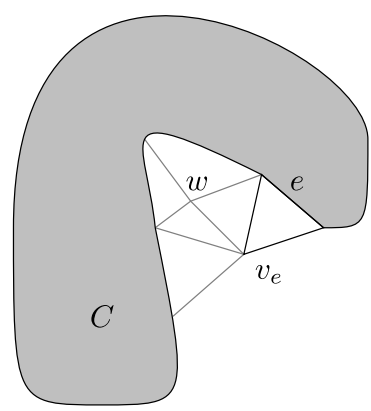

Fig. 13 Double happiness

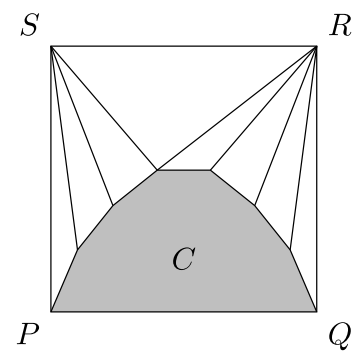

Note that Triangles $(C) \cup \Delta_{e}$ is contiguous, for each $e$. Thus $\Delta_{e}$ fails to be killable only if either $C \cup\left\{v_{e}\right\}$ contains at least three corners (and thus isn't a condition) or $C \cup\left\{v_{e}\right\}$ is separating.

Fix $e$ and suppose $C^{\prime}=C \cup\left\{v_{e}\right\}$ is separating. Then there is a loop $\gamma$ in the 1skeleton of $T$ that uses only vertices of $C^{\prime}$ and that encloses at least one vertex $w \notin C^{\prime}$. See Fig. 12. Now, note that any triangle $\Delta_{f}$ in the bounded region determined by $\gamma$ is killable (for $(T, C)$ ) unless $C \cup\left\{v_{f}\right\}$ is separating. By an innermost argument, not all such conditions can be separating. Thus $(T, C)$ has a killable triangle.

We may therefore assume that for each $e, C \cup\left\{v_{e}\right\}$ is non-separating. Now, if $C$ contains at most one corner, then $C \cup\left\{v_{e}\right\}$ contains at most two corners and thus $\Delta_{e}$ is killable. So, suppose $C$ contains two corners.

If these corners are not adjacent, then $\mathcal{T}$ is linear of type $\mathrm{D}$, so we may assume that the corners are adjacent, say $P$ and $Q$. We need to show that for some $e \subset \partial C$ other than $e=P Q, v_{e}$ is not a corner.

But if $v_{e}$ is a corner for each such $e$, it follows that either all vertices of $\partial C$ are connected by an edge to $R$ (or $S$ ), in which case $T$ contains a diagonal and is linear of type $\mathrm{D}$, or else there is a vertex $v$ of $\partial C$ that is connected by edges to both $R$ and $S$, in which case $\mathcal{T}$ is linear of type $\mathrm{X}$. See Fig. 13.

Armed with these two lemmas, we now present an algorithm that conjecturally computes $\operatorname{deg} \mathcal{T}$.

Algorithm 16 Let the triangulation $\mathcal{T}=(T, C)$ be given, with $|\partial T|=4$ and $C$ nonseparating. 
(i) If $\mathcal{T}$ has mosquitos, eliminate them by re-triangulating (as in the proof of Theorem 5).

(ii) If $T$ has subdivisions, delete them to obtain $\mathcal{T}^{\prime}$ as in Lemma 13. Recursively set $d(\mathcal{T})=d\left(\mathcal{T}^{\prime}\right)$ and stop.

(iii) Let $\Delta=x y z$ be a killable triangle. If none exists, set $d(\mathcal{T})=1$ and stop.

(iv) If $C=\emptyset$, set $\mathcal{T}^{\prime}=(T,\{x, y, z\})$, recursively set $d(\mathcal{T})=d\left(\mathcal{T}^{\prime}\right)$, and stop.

(v) If $C \neq \emptyset$, then $\Delta$ intersects $C$ in an edge $e$. Set $\mathcal{T}^{\prime}=T / e$ and $\mathcal{T}^{\prime \prime}=(T$, $C \cup\{x, y, z\})$.

(vi) If $\mathcal{T}^{\prime \prime}$ has mosquitos then discard it, set $d(\mathcal{T})=d\left(\mathcal{T}^{\prime}\right)$, and stop.

(vii) If $\mathcal{T}^{\prime \prime}$ has no mosquitos then set $d(\mathcal{T})=d\left(\mathcal{T}^{\prime}\right)+d\left(\mathcal{T}^{\prime \prime}\right)$.

Remark Step (iii) often allows for a choice of killable triangle $\Delta$. Different choices can lead to different outputs; see Sect. 6 for examples of this. Thus $d(\mathcal{T})$ is not a well-defined integer. However, we have the following result.

Theorem 17 Any output $d(\mathcal{T})$ computed by Algorithm 16 satisfies $d(\mathcal{T}) \leq \operatorname{deg}(\mathcal{T})$.

Proof We induct on the dimension of $V=V(\mathcal{T})$. Fix $\mathcal{T}=(T, C)$. Eliminating mosquitos doesn't change $V$ (or the degree), so we may assume $\mathcal{T}$ has no mosquitos. If $T$ has a subdivision then we can delete it by Lemma 13, and then by induction we are done. So we may also assume that $T$ has no subdivisions. Next, if $\mathcal{T}$ has no killable triangles then the algorithm sets $d(\mathcal{T})=1$, and, by Lemma 15, $\mathcal{T}$ is linear of type $\mathrm{D}$ or $\mathrm{X}$, $\operatorname{so} \operatorname{deg}(\mathcal{T})=1$ and again we are done. We may therefore assume $\mathcal{T}$ has a killable triangle $\Delta=x y z$.

Consider the variety $\widetilde{V}=V \cap\left\{A_{\Delta}=0\right\}$. If $C=\emptyset$ then $\widetilde{V}=V\left(\mathcal{T}^{\prime}\right)$ where $\mathcal{T}^{\prime}=$ $(T,\{x, y, z\})$, so $\widetilde{V}$ is irreducible by Theorem 3 . Thus the polynomial $\left.p(\mathcal{T})\right|_{\Delta=0}$ has the unique irreducible factor $p\left(\mathcal{T}^{\prime}\right)$. So $\operatorname{deg} p(\mathcal{T}) \geq \operatorname{deg} p\left(\mathcal{T}^{\prime}\right) \geq d\left(\mathcal{T}^{\prime}\right)=d(\mathcal{T})$ (the last inequality by induction, the last equality by the algorithm).

If $C \neq \emptyset$, then there is an edge $x y$ in both $\Delta$ and $\partial C$. In this case, a generic point $q$ of $\widetilde{V}$ is in the image of the area map, and so there is a drawing $\rho$ realizing the areas $q$. Note that either $\rho(x)=\rho(y)$ or the four points $\rho(x), \rho(y), \rho(w), \rho(z)$ are collinear (where $w$ and $z$ are the vertices of $T$ that are adjacent to both $x$ and $y$ ). Thus $q$ is in either $V^{\prime}=V\left(\mathcal{T}^{\prime}\right)$ or $V^{\prime \prime}=V\left(\mathcal{T}^{\prime \prime}\right)$ (where we think of these as being subsets of $V$ in the obvious way). Hence the only possible components of $\widetilde{V}$ are $V^{\prime}$ and $V^{\prime \prime}$, and each of these is a component if and only if it has codimension $1 \mathrm{in} V$.

To test whether $V^{\prime}$ and $V^{\prime \prime}$ are components, we use Theorem 5. Since $\operatorname{dim} X^{\prime}=$ $\operatorname{dim} X^{\prime \prime}=\operatorname{dim} X-1$, the only way that $V^{\prime}$ or $V^{\prime \prime}$ could fail to have codimension 1 in $V$ is if the corresponding triangulation has mosquitos. Lemma 18 (below) asserts that $\mathcal{T}^{\prime}$ has no mosquitos, so $V^{\prime}$ is a component of $\widetilde{V}$. If $\mathcal{T}^{\prime \prime}$ has mosquitos we discard it; otherwise $V^{\prime \prime}$ is also a component of $\widetilde{V}$. In the case that both $V^{\prime}$ and $V^{\prime \prime}$ are components of $\widetilde{V}$, we note that $V^{\prime} \neq V^{\prime \prime}$, since it is easy to construct points of one that are not on the other. Therefore, using the induction hypothesis, if $\widetilde{V}$ has just one component, then $\operatorname{deg} p=\left.\operatorname{deg} p\right|_{\Delta=0} \geq \operatorname{deg} \mathcal{T}^{\prime} \geq d\left(\mathcal{T}^{\prime}\right)=d(\mathcal{T})$, whereas if $\widetilde{V}$ has two components then $\operatorname{deg} p=\left.\operatorname{deg} p\right|_{\Delta=0} \geq \operatorname{deg} p^{\prime}+\operatorname{deg} p^{\prime \prime} \geq d\left(\mathcal{T}^{\prime}\right)+$ $d\left(\mathcal{T}^{\prime \prime}\right)=d(\mathcal{T})$. 
Lemma 18 Let $\mathcal{T}=(T, C)$ be a triangulation with no mosquitos and with $C$ nonempty and non-separating. Let $\Delta=x y z$ be a killable triangle, where $\{x, y\} \subset C$. Let $e=x y$ and let $\mathcal{T}^{\prime}=T / e$. Then $\mathcal{T}^{\prime}$ has no mosquitos.

Proof If $C / e=\emptyset$ then certainly $\mathcal{T}^{\prime}$ has no mosquitos. Otherwise $C / e$ is the image of $C$ under the identification $x=y$. Suppose $v \in \operatorname{Vertices}(T / e)$ is a mosquito in $\mathcal{T}^{\prime}$. Then $v$ and all its neighbors in $T / e$ are vertices of $C / e$. Since $x$ and $y$ are both in $C$, this implies that $v$ and all its neighbors in $T$ are vertices of $C$. But $\mathcal{T}$ has no mosquitos, so this is a contradiction.

Remark In a recursive computation of $d(\mathcal{T})$, if it happens that every time we intersect a variety with a hyperplane, the components of intersection have multiplicity 1 , then each inequality in the above proof is an equality, and we have $d(\mathcal{T})=\operatorname{deg}(\mathcal{T})$.

Conjecture 1 For any $\mathcal{T}$, it is possible to run Algorithm 16 in such a way that at each step, every component has geometric multiplicity one.

If the conjecture is true, then by killing the triangles in the specified order, the algorithm will produce the degree of $p(\mathcal{T})$.

As an approach to this conjecture, we point out that if there are any nonsingular points of $V$ in the intersection $V \cap\left\{A_{\Delta}=0\right\}$, then in most cases killing the triangle $\Delta$ results in components of multiplicity one. To see this, one can use a simple differential geometry argument we call "busting through." The idea is to pick a nonsingular point $x$ of $V \cap\left\{A_{\Delta}=0\right\}$ and construct a curve $\gamma \subset V$ through $x$ that is not tangent to $\left\{A_{\Delta}=0\right\}$. The existence of $\gamma$ shows that $V$ itself is not tangent to $\left\{A_{\Delta}=0\right\}$, and so the multiplicity of intersection of any component must be one. However, examples in Sect. 8 show that it is possible for $V \cap\left\{A_{\Delta}=0\right\}$ to consist entirely of singular points of $V$.

\section{Consequences of the Degree Algorithm}

Although we cannot guarantee that Algorithm 16 returns deg $\mathcal{T}$, we are nevertheless able to use the algorithm to deduce some results about the degree. Specifically, in case $C=\emptyset$ we give a general lower bound for $\operatorname{deg} \mathcal{T}$ in terms of $|\operatorname{Vertices}(T)|$, and for arbitrary $C$ we characterize triangulations $\mathcal{T}$ with $\operatorname{deg} \mathcal{T}=1$. The latter is similar to a result in [7].

Our first corollary requires a graph-theoretic lemma.

Lemma 19 Let $T$ be a simplicial complex homeomorphic to a disk, with $|\partial T|=4$ and with at least one vertex in the interior. Suppose T has no subdivisions. Then there is an edge $e=x y$ of $T$ such that $\{x, y\} \not \subset \partial T$ and $T / e$ has no subdivisions.

Proof We call a 4-cycle $\gamma$ in the 1-skeleton of $T$ bad if $\gamma$ encloses at least one vertex of $T$ (equivalently, if $\gamma$ encloses more than 2 triangles). Note that if the 4 cycle $w x y z$ is bad then neither $w y$ nor $x z$ is an edge of $T$, since $T$ is assumed to have no subdivisions. 
Fig. 14 The edge $e$ is part of a bad 4-cycle

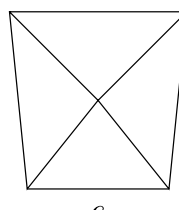

$T$

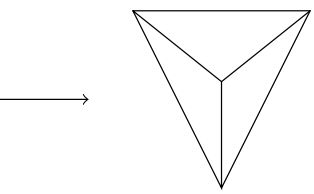

$T / e$

Fig. 15 The edge $e$ is not part of a bad 4-cycle

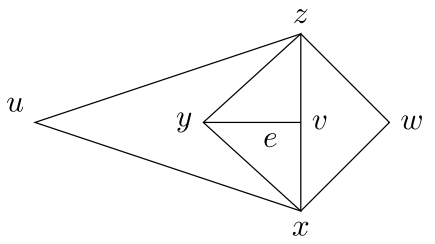

Let $e=x y$ be a non-boundary edge. If $T / e$ contains a subdivision, then there must be a bad 4-cycle $\gamma$ including the edge $e$. So it suffices to show that there is an edge $e$ that is not part of any bad 4-cycle. See Fig. 14.

For bad 4-cycles $\gamma, \gamma^{\prime}$ we say $\gamma \leq \gamma^{\prime}$ if every triangle enclosed by $\gamma$ is also enclosed by $\gamma^{\prime}$. Fix a bad 4-cycle $\gamma=w x y z$ that is minimal with respect to this partial order. Let $v$ be a vertex enclosed by $\gamma$, and let $e_{0}$ be an edge emanating from $v$.

If $e_{0}$ is not part of a bad 4-cycle, then we are done, so assume $e_{0}$ is part of a bad 4-cycle $\beta$. Since $\gamma$ is minimal, $\beta$ must enclose a triangle not enclosed by $\gamma$. Thus $\beta$ must intersect $\gamma$, and the four vertices of $\beta$ must be $v$, two of $w, x, y, z$, and a vertex $u$ that is outside $\gamma$. Since $\beta$ is bad, neither of its diagonals is an edge of $T$, so the set of vertices in $\beta \cap \gamma$ must be either $\{w, y\}$ or $\{x, z\}$. Without loss of generality, suppose $\beta=v x u z$. Moreover, exactly one of $w, y$ is inside $\beta$; suppose it is $y$. See Fig. 15.

Now, consider the 4-cycle $\alpha=v x y z$. By minimality of $\gamma, \alpha$ does not enclose a vertex. So $\alpha$ encloses exactly two triangles, and it follows that one of the diagonals of $\alpha$ is an edge of $T$. But $x z$ is not an edge of $T$, because $\gamma$ is bad. So $v y$ is an edge of $T$. We will show that $e=v y$ is not part of any bad 4-cycle.

Suppose there is a bad 4-cycle $\delta=v y a b$. It is impossible for $a$ to coincide with $x$, because the edge $v x$ would be a diagonal of the bad 4-cycle $v y x b$. Likewise $a \neq z$ and $x \neq b \neq z$. Thus, since $v$ is enclosed by $\gamma$ and $y$ is enclosed by $\beta$, the only possibility is $a=u$ and $b=w$.

Now, the 4-cycle $\delta=v y u w$ separates $x$ from $z$ in $T$, and thus must enclose exactly one of these vertices. But if $\delta$ encloses $x$, then $T$ contains the subdivision $w z u$, and if $\delta$ encloses $z$ then $T$ contains the subdivision $w x u$. This contradiction shows that $e$ satisfies the conclusion of the lemma.

Corollary 20 Let $\mathcal{T}=(T, \emptyset)$ be a triangulation of a square with $k$ interior vertices and no subdivisions. Then $\operatorname{deg} \mathcal{T} \geq k$.

Example 3 in the next section shows that this bound is sharp for all $k$. 
Proof We proceed by induction; the conclusion is true if $k \leq 1$. Suppose $k \geq 2$.

By Lemma 19, there is a non-boundary edge $e=x y$ such that $T / e$ has no subdivisions. As $C=\emptyset$, the edge $e$ is good. We run Algorithm 16 as follows. Let $w, z$ be the two vertices that are adjacent to both $x$ and $y$. Observe that the triangle wxy is killable. We first kill $w x y$ and obtain $\mathcal{T}^{\prime}=(T,\{w, x, y\})$ with $d\left(\mathcal{T}^{\prime}\right)=d(\mathcal{T})$. In $\mathcal{T}^{\prime}$, the triangle $x y z$ is killable, so we kill it, giving triangulations $\mathcal{T}^{\prime \prime}=\mathcal{T} / e$ and $\mathcal{T}^{\prime \prime \prime}=(T,\{w, x, y, z\})$.

Now, $\mathcal{T}^{\prime \prime}$ has $k-1$ interior vertices, no condition (hence no mosquitos), and by our choice of $e$, no subdivisions. Thus by induction we have $\operatorname{deg}\left(\mathcal{T}^{\prime \prime}\right) \geq k-1$. Also, since $T$ has no subdivisions, the condition in $\mathcal{T}^{\prime \prime \prime}$ is not big enough to create a mosquito, and so $d\left(\mathcal{T}^{\prime \prime \prime}\right) \geq 1$. Thus $\operatorname{deg} \mathcal{T} \geq \operatorname{deg}\left(\mathcal{T}^{\prime \prime}\right)+\operatorname{deg}\left(\mathcal{T}^{\prime \prime \prime}\right) \geq k$.

We need one more lemma before we characterize those triangulations $\mathcal{T}$ with $\operatorname{deg} \mathcal{T}=1$.

Lemma 21 Let $\mathcal{T}=(T, C)$ be a triangulation with no mosquitos and with $C$ nonseparating. Suppose $\Delta=x y z$ is a killable triangle with $\{x, y\} \subset C$. Let $\mathcal{T}^{\prime \prime}=(T, C \cup$ $\{z\})$. If $\mathcal{T}^{\prime \prime}$ has a mosquito then either $x$ or $y$ is a mosquito of $\mathcal{T}^{\prime \prime}$.

Proof Let $m$ be a mosquito of $\mathcal{T}^{\prime \prime}$; we may assume $m \notin\{x, y\}$. Thus $m \in C$ and since $\mathcal{T}$ has no mosquitos, there must be an edge $m z$ in $T$. Let $\alpha$ be an embedded path in $\partial C$ from $m$ to $x$ such that $\alpha$ does not go through $y$, and let $\beta$ be the union of $\alpha$ and the path $m z x$. Thus $\beta$ is an embedded loop in $T$.

Suppose neither $x$ nor $y$ is a mosquito in $\mathcal{T}^{\prime \prime}$. Then there are neighbors $s, t$ of $x, y$ such that $s \neq z \neq t$ and $s, t \notin C$. See Fig. 16. Consider the path $\gamma=s x y t$. Note that $\gamma$ intersects $\beta$ in exactly one point, namely $x$. This intersection is transverse, since the edges coming out of $x$ occur in the cyclic order $y, z, s, \alpha$. Thus exactly one of $s, t$ is enclosed by $\beta$. But this means that $C \cup\{z\}$ fails to be non-separating, contradicting the assumption that $\Delta$ is killable.

Corollary 22 Let $\mathcal{T}=(T, C)$ be a triangulation of a square with $C$ non-separating. Then $\operatorname{deg} \mathcal{T}=1$ if and only if $\mathcal{T}$ is linear of type $D$ or $X$.

Proof We have already seen that triangulations that are linear of type D or $\mathrm{X}$ have degree 1 . To prove the converse, we will use induction on the dimension of $V(\mathcal{T})$.

Fig. 16 The loop $\beta$ must enclose either $s$ or $t$

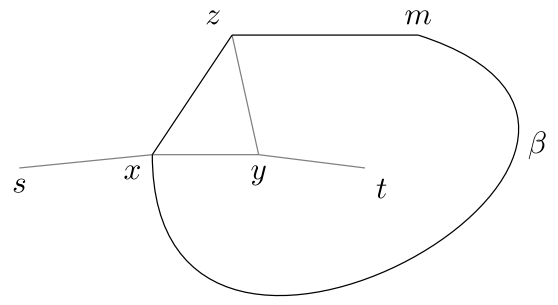


Let $\mathcal{T}=(T, C)$ be a triangulation and suppose $\operatorname{deg} \mathcal{T}=1$. We wish to show that $\mathcal{T}$ is linear of type D or X. If not, then by Lemma 15, when we run Algorithm 16 we will find a killable triangle $\Delta=x y z$.

Consider first the case that $C=\emptyset$. Then $\operatorname{deg} \mathcal{T}^{\prime}=\operatorname{deg} \mathcal{T}=1$ where $\mathcal{T}^{\prime}=$ $(T,\{x, y, z\})$. Since $\operatorname{dim} V^{\prime}<\operatorname{dim} V$, it follows by induction that $\mathcal{T}^{\prime}$ is linear of type $\mathrm{D}$ or $\mathrm{X}$. But two vertices are joined in $\mathcal{T}^{\prime}$ if and only if they are joined in $\mathcal{T}$; thus $\mathcal{T}$ is also linear of type $\mathrm{D}$ or $\mathrm{X}$. (Note that the case $C=\emptyset$ also follows from Corollary 20.)

Now suppose that $C \neq \emptyset$. Let $\mathcal{T}^{\prime}$ and $\mathcal{T}^{\prime \prime}$ be as in Algorithm 16. It follows from the proof of Theorem 17 that $\operatorname{deg} \mathcal{T}^{\prime}=1$ and that $\mathcal{T}^{\prime \prime}$ contains at least one mosquito. By induction, $\mathcal{T}^{\prime}$ is linear of type $\mathrm{D}$ or $\mathrm{X}$.

We first consider the case that $\mathcal{T}^{\prime}$ is linear of type D. Thus two opposite corners, say $P$ and $R$, are joined in $\mathcal{T}^{\prime}$. If $P$ and $R$ are joined in $\mathcal{T}$, we are done, so assume the opposite. Then it must be the case that either $x$ or $y$ is a corner, say $x=R$, so that $y$ is connected to both $P$ and $R$. Now, since $\mathcal{T}^{\prime}$ has at least one mosquito, Lemma 21 implies that $y$ must be a mosquito in $\mathcal{T}^{\prime \prime}$. In particular, this implies $P, R \in C$ and so $P$ and $R$ are joined in $\mathcal{T}$ after all.

Next suppose $\mathcal{T}^{\prime}$ is linear of type X. Let $v$ be a vertex of $\mathcal{T}^{\prime}$ that is joined to all four corners. If $v$ is joined to all four corners in $\mathcal{T}$, then we are done, so assume the opposite. It follows that $v$ must be the image of $x$ (and $y$ ) under the contraction $\operatorname{Vertices}(T) \rightarrow \operatorname{Vertices}(T / e)$. Again, since $\mathcal{T}^{\prime \prime}$ has at least one mosquito, Lemma 21 implies that either $x$ or $y$ is a mosquito of $\mathcal{T}^{\prime \prime}$; suppose it is $x$. Therefore, every neighbor of $x$ other than $z$ is in $C$. We now claim that $y$ is joined to all four corners in $\mathcal{T}$, and hence $\mathcal{T}$ is linear of type X. To see this, consider any corner $w$. If $w=z$ then there is an edge from $w$ to $y$. Thus we can assume $w \neq z$. Since $w$ is joined to $v$ in $\mathcal{T}^{\prime}$, it must be that $w$ is joined to either $x$ or $y$ in $\mathcal{T}$. But if $w$ is joined to $x$ in $\mathcal{T}$, then $w \in C$, because $w \neq z$ and every neighbor of $x$ in $\mathcal{T}$ other than $z$ is in $C$. Thus either way, $w$ is joined to $y$ in $\mathcal{T}$.

\section{Examples}

Example 2 Let $\mathcal{T}=\mathcal{T}_{2}=\left(T_{2}, \emptyset\right)$ be the triangulation in Fig. 17. Using elementary algebra, one can easily compute that $p(\mathcal{T})=(A+C+E)^{2}-4 A C-(B+D+F)^{2}+$ $4 D F$; see Example 3 for a more general computation. In particular, $\operatorname{deg}(\mathcal{T})=2$. One way to run the algorithm on $\mathcal{T}$ is the following. Note that $T$ has no subdivisions. We first kill $B$ to obtain $\mathcal{T}^{1}=(T,\{Q, x, y\}$ ) (where $x$ and $y$ are the names of the two interior vertices), and we set $d(\mathcal{T})=d\left(\mathcal{T}^{1}\right)$.

Next kill $E$, obtaining $\mathcal{T}^{2}=\left(\left.T\right|_{x=y}, \emptyset\right)$ and $\mathcal{T}^{3}=(T,\{Q, x, y, S\})$. Each of these has the correct dimension, so we set $d\left(\mathcal{T}^{1}\right)=d\left(\mathcal{T}^{2}\right)+d\left(\mathcal{T}^{3}\right)$. After killing one more triangle of $\mathcal{T}^{2}$, there will be no more killable triangles, and the algorithm will return $d\left(\mathcal{T}^{2}\right)=1$, which, in fact, is the correct degree since $p\left(\mathcal{T}^{2}\right)=A-C-D+F$. Meanwhile, $\mathcal{T}^{3}$ already has no killable triangles, so the algorithm returns $d\left(\mathcal{T}^{3}\right)=1$, and again this is the correct degree since $p\left(\mathcal{T}^{3}\right)=A-C+D-F$.

Thus the result of the algorithm is that $d(\mathcal{T})=d\left(\mathcal{T}^{1}\right)=d\left(\mathcal{T}^{2}\right)+d\left(\mathcal{T}^{3}\right)=$ $1+1=2$, and in this case the algorithm produces the correct answer, $d(\mathcal{T})=$ $\operatorname{deg}(\mathcal{T})=2$. 
Fig. 17 The top triangulation, $\mathcal{T}_{2}$, has degree 2 . Its Monsky polynomial is

$(A+C+E)^{2}-4 A C-$ $(B+D+F)^{2}+4 D F$
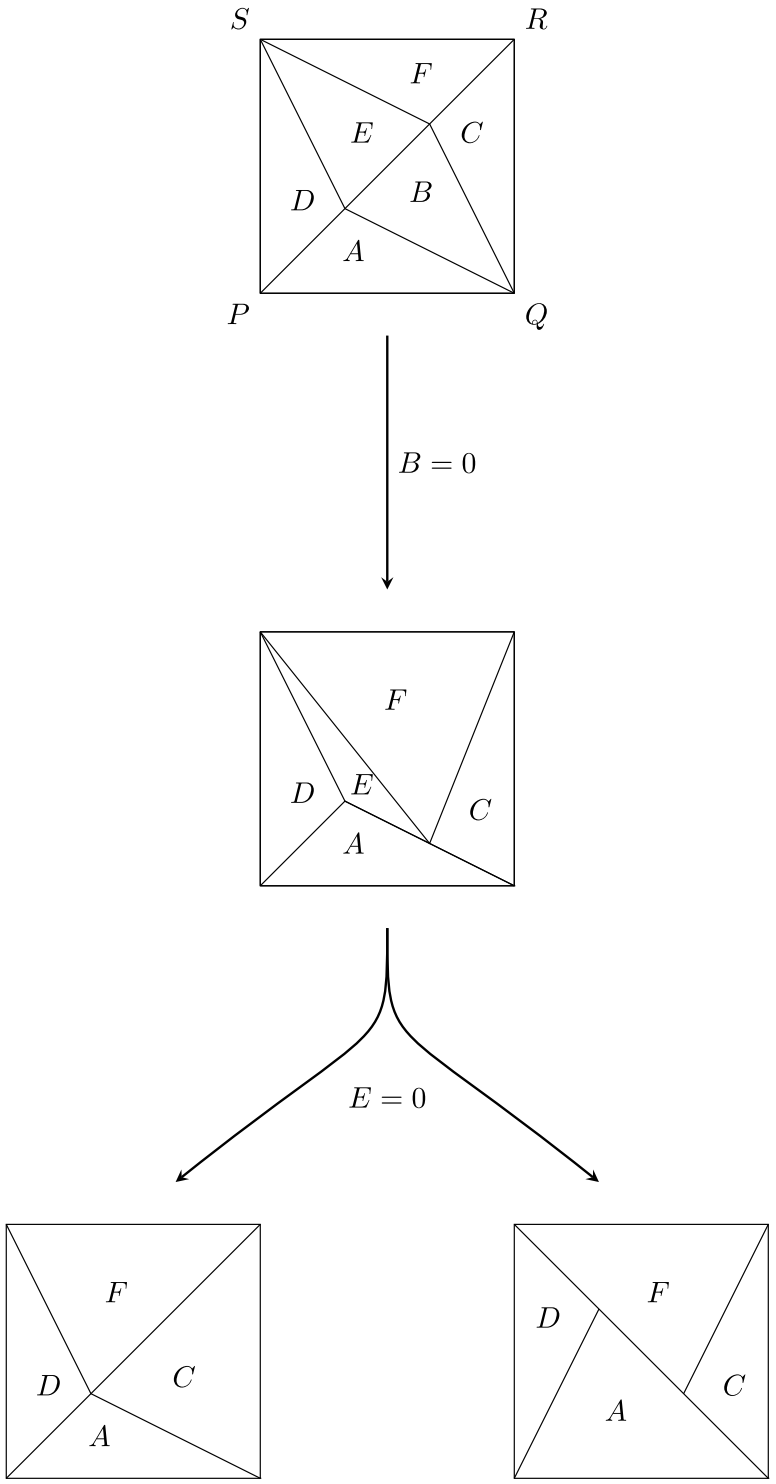

Note that $p\left(\mathcal{T}^{1}\right)=\left.p(\mathcal{T})\right|_{B=0}$ is irreducible; this is why $\operatorname{deg}\left(\mathcal{T}^{1}\right)=\operatorname{deg}(\mathcal{T})$. On the other hand, $\left.p\left(\mathcal{T}^{1}\right)\right|_{E=0}=(A+C)^{2}-4 A C-(D+F)^{2}+4 D F$ factors as $(A-$ $C-D+F)(A-C+D-F)$. These factors are the polynomials associated to the combinatorial "factors" $\mathcal{T}^{2}$ and $\mathcal{T}^{3}$ of $\left.\mathcal{T}^{1}\right|_{E=0}$.

Up to isomorphism, $T_{2}$ is the only triangulation with two interior vertices and no subdivisions. Thus, by Corollary $20, \mathcal{T}_{2}$ is the only triangulation with $C=\emptyset$ and with no subdivisions that satisfies $\operatorname{deg} \mathcal{T}=2$. 
Fig. 18 The triangulation $T_{n}$

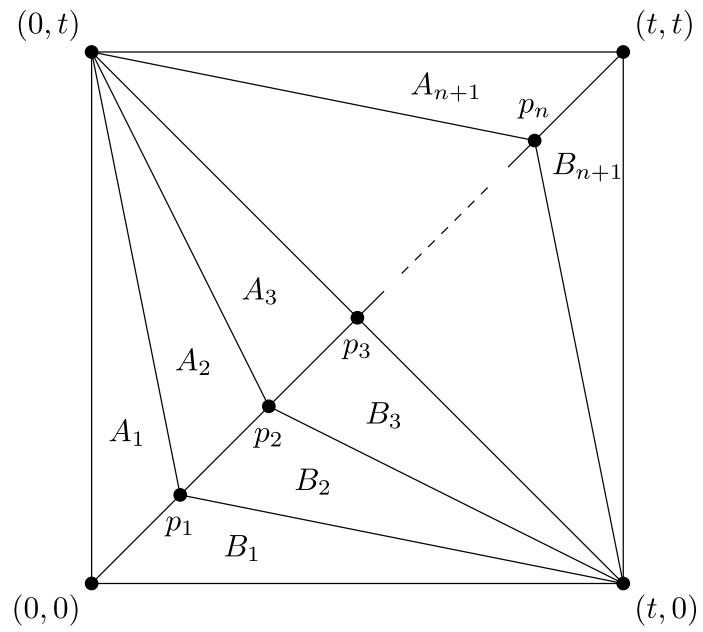

Example 3 (The diagonal case) Examples 1 and 2 begin an infinite sequence of triangulations $\mathcal{T}_{n}=\left(T_{n}, \emptyset\right)$ that we refer to as the "diagonal case." For $n \geq 1$, the triangulation $T_{n}$ has a total of $n+4$ vertices, with the $n$ interior vertices arranged along a diagonal of the square. Each is connected to its neighbors and to the two opposite corners of the square, as in Fig. 18. (In viewing this figure, note that for $1 \leq i \leq n$ the $p_{i}$ are not constrained to be on the diagonal of the square.)

We will compute the polynomial $p\left(\mathcal{T}_{n}\right)$ for each $n$ using a straightforward elimination of variables. In particular, we will show that $\operatorname{deg}\left(\mathcal{T}_{n}\right)=n$.

For convenience we illustrate with drawings of a square in the plane with coordinates $(0,0),(t, 0),(t, t)$, and $(0, t)$. We label the $n$ interior points $p_{i}=\left(x_{i}, y_{i}\right)$ (in "increasing order" as in Fig. 18), and let $p_{0}=(0,0)$ and $p_{n+1}=(t, t)$. Then $\mathcal{T}_{n}$ has edges connecting $p_{i}$ to $p_{i+1}$, for $i=0,1, \ldots, n$, and also connecting each $p_{i}$ to both corners $(0, t)$ and $(t, 0)$.

For the areas we use the following notation: for $1 \leq i \leq n+1$, let $A_{i}$ denote twice the area of the triangle with vertices $\left\{(0, t), p_{i-1}, p_{i}\right\}$ and let $B_{i}$ denote twice the area of the triangle with vertices $\left\{(t, 0), p_{i}, p_{i-1}\right\}$. This accounts for all $2 n+2$ triangles.

It is convenient to define the linear forms

$$
\begin{aligned}
\sigma_{k} & :=\sum_{i=1}^{k}\left(A_{i}+B_{i}\right), \\
\bar{\sigma}_{k} & :=\sum_{i=k+1}^{n+1}\left(A_{i}+B_{i}\right)=2 t^{2}-\sigma_{k} .
\end{aligned}
$$

We allow $0 \leq k \leq n+1$, i.e., $\sigma_{0}=0=\bar{\sigma}_{n+1}$ and $\bar{\sigma}_{0}=2 t^{2}=\sigma_{n+1}$.

We now begin the elimination of variables. In other words, beginning with the formulas expressing the $A_{i}$ and $B_{i}$ in terms of $x_{i}, y_{i}$, and $t$, we systematically eliminate $x_{i}, y_{i}$, and $t$. 
Here is the starting point:

$$
\begin{aligned}
A_{i} & =\left|\begin{array}{ccc}
1 & 1 & 1 \\
0 & x_{i-1} & x_{i} \\
t & y_{i-1} & y_{i}
\end{array}\right|=x_{i-1} y_{i}-x_{i} y_{i-1}+t\left(x_{i}-x_{i-1}\right) \\
B_{i} & =\left|\begin{array}{ccc}
1 & 1 & 1 \\
t & x_{i} & x_{i-1} \\
0 & y_{i} & y_{i-1}
\end{array}\right|=-x_{i-1} y_{i}+x_{i} y_{i-1}+t\left(y_{i}-y_{i-1}\right)
\end{aligned}
$$

Lemma 23 For each $0 \leq k \leq n+1$,

$$
y_{k}=\frac{\sigma_{k}}{t}-x_{k} .
$$

Proof It is clear both from the above formulas and from the picture (imagining a segment drawn from the origin to $\left.p_{k}\right)$ that $t\left(x_{k}+y_{k}\right)=\sigma_{k}$.

Using the lemma, we easily eliminate all $y$ 's. So, on to the $x$ 's. Substituting for the $y$ 's in the formula for $B_{k}$ yields

$$
\begin{aligned}
B_{k} & =-x_{k-1} y_{k}+x_{k} y_{k-1}+t\left(y_{k}-y_{k-1}\right) \\
& =-x_{k-1}\left(\frac{\sigma_{k}}{t}-x_{k}\right)+x_{k}\left(\frac{\sigma_{k-1}}{t}-x_{k-1}\right)+\left(\sigma_{k}-\sigma_{k-1}\right)-t x_{k}+t x_{k-1} \\
& =x_{k}\left(\frac{\sigma_{k-1}}{t}-t\right)-x_{k-1}\left(\frac{\sigma_{k}}{t}-t\right)+A_{k}+B_{k},
\end{aligned}
$$

where on the last line we used the fact that $\sigma_{k}-\sigma_{k-1}=A_{k}+B_{k}$. Solving for $x_{k}$ gives

$$
x_{k}=\frac{1}{t} \frac{\left(t x_{k-1}\right)\left(\sigma_{k}-t^{2}\right)-t^{2} A_{k}}{\left(\sigma_{k-1}-t^{2}\right)} .
$$

(We have deliberately chosen to write a factor of $1 / t$ in front, even though it could be canceled.) Now, by (1) we rewrite $\sigma_{i}-t^{2}=\left(\sigma_{i}-\bar{\sigma}_{i}\right) / 2$ and $t^{2}=\bar{\sigma}_{0} / 2=-\left(\sigma_{0}-\right.$ $\left.\bar{\sigma}_{0}\right) / 2$, thus obtaining

$$
x_{k}=\frac{1}{t} \frac{\left(t x_{k-1}\right)\left(\sigma_{k}-\bar{\sigma}_{k}\right)+\left(\sigma_{0}-\bar{\sigma}_{0}\right) A_{k}}{\left(\sigma_{k-1}-\bar{\sigma}_{k-1}\right)} .
$$

We now have $x_{k}$ written in terms of $x_{k-1}$, so we may recursively eliminate the $x$ 's.

For shorthand we will use the notation $L_{k}$ for the linear form $\sigma_{k}-\bar{\sigma}_{k}$, and

$$
L_{k_{1} \cdots k_{j}}=\prod_{i=1}^{j} L_{k_{i}}=\prod_{i=1}^{j}\left(\sigma_{k_{i}}-\bar{\sigma}_{k_{i}}\right) .
$$

So our expression for $x_{k}$ becomes

$$
x_{k}=\frac{1}{t} \frac{\left(t x_{k-1}\right) L_{k}+A_{k} L_{0}}{L_{k-1}} .
$$


Lemma 24 For $1 \leq k \leq n+1$, we have

$$
x_{k}=\frac{1}{t} \frac{p_{k}}{q_{k}}
$$

where $p_{k}$ is the degree $k$ polynomial

$$
p_{k}=L_{01 \cdots k}\left(\frac{A_{1}}{L_{01}}+\frac{A_{2}}{L_{12}}+\cdots+\frac{A_{k}}{L_{k-1, k}}\right)
$$

and $q_{k}$ is the degree $k-1$ polynomial $q_{k}=L_{12 \cdots(k-1)}$. (In case $k=1, q_{1}$ is the empty product 1.)

Proof When $k=1$ the claim is that $x_{1}=A_{1} / t$, which is clearly true. The rest follows by induction from (5). Specifically, if $x_{k-1}=\frac{1}{t} \frac{p_{k-1}}{q_{k-1}}$ then plugging into (5) gives the recursions

$$
q_{k}=q_{k-1} L_{k-1}
$$

and

$$
p_{k}=p_{k-1} L_{k}+A_{k} L_{0} q_{k-1}
$$

The formula for $q_{k}$ follows immediately, and the formula for $p_{k}$ is easily verified.

The preceding lemma is valid for $k=n+1$, so we now have an explicit expression for $x_{n+1}$ in terms of $t$ and the $A_{i}$ and $B_{i}$. But $x_{n+1}=t$. This gives the relation $t=$ $x_{n+1}=\frac{1}{t} \frac{p_{n+1}}{q_{n+1}}$, which we write as $-L_{0} q_{n+1}=2 p_{n+1}$ (recalling that $-L_{0}=2 t^{2}$ ). Plugging in for $q_{n+1}$ and $p_{n+1}$ from the preceding lemma gives

$$
\begin{aligned}
-L_{01 \cdots n} & =2 L_{01 \cdots(n+1)}\left(\frac{A_{1}}{L_{01}}+\frac{A_{2}}{L_{12}}+\cdots+\frac{A_{n+1}}{L_{n, n+1}}\right) \\
& =2\left(A_{1} L_{23 \cdots(n+1)}+A_{2} L_{34 \cdots n+1,0}+\cdots+A_{n+1} L_{01 \ldots(n-1)}\right) .
\end{aligned}
$$

This is a homogeneous degree $n+1$ polynomial relation among the areas $A_{i}, B_{i}$, and the coefficients are integers with no common factor. Thus, by Theorem 10 , the polynomial $p\left(\mathcal{T}_{n}\right)$ must be an irreducible factor of (6). Note that (6) itself is not irreducible: both sides of the above equation contain a (linear) factor of $-L_{0}=L_{n+1}=$ $\sum\left(A_{i}+B_{i}\right)$. Since there exist nondegenerate drawings of $T_{n}$ (i.e., drawings in which $t \neq 0$ and hence the total area is nonzero), this linear factor is not equal to $p\left(\mathcal{T}_{n}\right)$. If we factor this out, we are left with a degree $n$ polynomial; we next argue that this must be equal to $p\left(\mathcal{T}_{n}\right)$.

One way to write the degree $n$ relation is to cancel $-L_{0}$ from the left side against $L_{n+1}$ from every term on the right except the last, and $L_{0}$ from the remaining term. This puts the remaining polynomial in the form recorded in the following theorem. 
Theorem 25 Let $n \geq 1$ and let $\mathcal{T}_{n}=\left(T_{n}, \emptyset\right)$, where $T_{n}$ is the triangulation illustrated in Fig. 18. The polynomial $p\left(\mathcal{T}_{n}\right)$ is equal to

$$
L_{12 \cdots n}-2\left(A_{1} L_{23 \cdots n}+A_{2} L_{34 \cdots n 0}+\cdots+A_{n} L_{01 \cdots(n-2)}-A_{n+1} L_{12 \cdots(n-1)}\right) .
$$

In particular, $\operatorname{deg}\left(\mathcal{T}_{n}\right)=n$.

(See Eqs. (1), (2), and (4) for the notation.) Note that the polynomial (8) has integer coefficients. By modifying CoCoA code originally written by D. Perkinson, we computed $p\left(\mathcal{T}_{n}\right)$ for $n=1,2,3,4,5$, and the CoCoA script produced this polynomial divided by $2^{n-1}$. For $n \geq 6$ the problem was too large for our laptops.

Proof We have shown that the areas $A_{i}, B_{i}$ satisfy (8). If one were to prove directly that this polynomial is irreducible, then it would follow that it equals $p\left(\mathcal{T}_{n}\right)$. However, this is unnecessary because Corollary 20 implies that the degree of $p\left(\mathcal{T}_{n}\right)$ is at least $n$, and so $(8)$ must be the polynomial $p\left(\mathcal{T}_{n}\right)$ (and it follows that (8) is irreducible).

The next family of examples illustrates two notable phenomena. First, we show that $\operatorname{deg}(\mathcal{T})$ can grow exponentially in the number of vertices of the triangulation. In a slightly different context, this was also observed in [7]. In that paper, the authors give a general upper bound $\operatorname{deg}(\mathcal{T}) \leq(t+1) 2^{t}$ where $t$ is the number of non-degenerate triangles in $\mathcal{T}$.

Second, we will see that when running Algorithm 16, it is possible to get arbitrarily large multiplicities of components.

Example 4 We define triangulations $T_{n, k}$ for all non-negative integers $n$ and $k$ with $k \leq \frac{n+1}{2}$. Roughly speaking, we form $T_{n, k}$ by starting with the diagonal case $T_{n}$ and "exploding" exactly $k$ length 2 segments along the diagonal into pairs of triangles. (In particular $T_{n, 0}=T_{n}$.) Precisely, $T_{n, k}$ has a total of $n+k+4$ vertices: the four corners $P=p_{0}, Q, R=p_{n+1}, S$; the $n$ vertices $p_{1}, \ldots, p_{n}$ arranged diagonally as before; and also "duplicates" $p_{1}^{\prime}, p_{3}^{\prime}, \ldots, p_{2 k-1}^{\prime}$ of $k$ of the diagonal vertices. The $2 n+2 k+2$ triangles of $T_{n, k}$ have the following vertex sets (see Fig. 19):

- $\left\{S, p_{i}, p_{i+1}\right\}$ for $i=0, \ldots, n$;

- $\left\{Q, p_{2 i-1}^{\prime}, p_{2 i-2}\right\}$ for $i=1, \ldots, k$;

- $\left\{Q, p_{2 i}, p_{2 i-1}\right\}$ for $i=1, \ldots, k$;

- $\left\{Q, p_{i+1}, p_{i}\right\}$ for $i=2 k, \ldots, n$;

- $\left\{p_{2 i-2}, p_{2 i-1}^{\prime}, p_{2 i-1}\right\}$ for $i=1, \ldots, k$;

- $\left\{p_{2 i}, p_{2 i-1}, p_{2 i-1}^{\prime}\right\}$ for $i=1, \ldots, k$.

Note that one obtains $T_{n}$ from $T_{n, k}$ by contracting each of the edges $p_{i} p_{i}^{\prime}$.

Theorem 26 For the triangulation $\mathcal{T}_{n, k}=\left(T_{n, k}, \emptyset\right)$, we have

$$
\operatorname{deg}\left(\mathcal{T}_{n, k}\right) \geq 2^{k-1}(2 n-k)
$$

Proof We proceed by induction on $k$, by running the algorithm. If $k=0$, the triangulation is the diagonal case and the result agrees with the previous example. 
Fig. 19 The exploded diagonal triangulation $T_{5,2}$

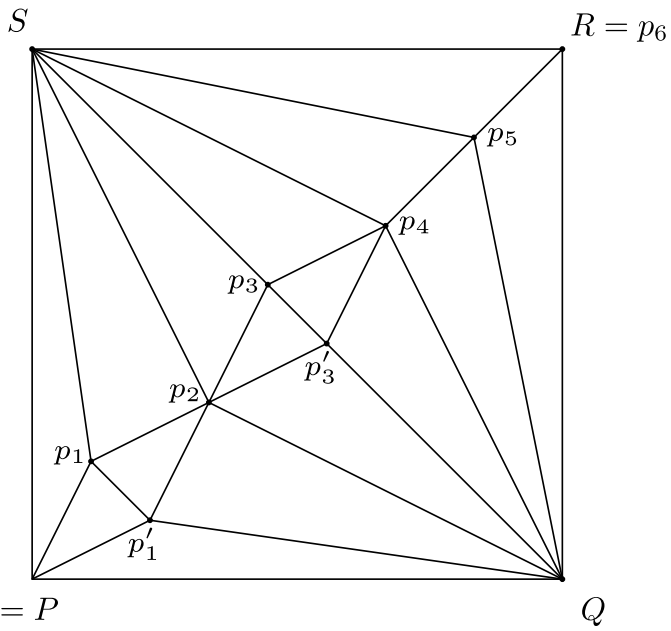

Suppose $k>0$. We first kill the triangle $\left\{p_{2 k}, p_{2 k-1}, p_{2 k-1}^{\prime}\right\}$. The result has a single component. We then kill the triangle $\left\{p_{2 k-2}, p_{2 k-1}^{\prime}, p_{2 k-1}\right\}$. The result has two components. One is obtained by contracting the edge $p_{2 k-1} p_{2 k-1}^{\prime}$; this triangulation is exactly $T_{n, k-1}$.

The other component, $\dot{\mathcal{T}}$, has a condition consisting of the four vertices

$$
\left\{p_{2 k-2}, p_{2 k-1}, p_{2 k-1}^{\prime}, p_{2 k}\right\} \text {. }
$$

We analyze this using the following

Useful trick Suppose $\mathcal{T}=(T, C)$ is a triangulation, where $C \supset\{w, x, y, z\}$ and $w x y$ and $x y z$ are triangles of $T$. Let $\hat{T}$ be the triangulation obtained from $T$ by replacing the triangles $w x y$ and $x y z$ with triangles $w x z$ and wyz, and let $\hat{\mathcal{T}}=(\hat{T}, C)$. Then $p(\mathcal{T})=p(\hat{\mathcal{T}})$.

The trick works because $X(\mathcal{T})=X(\hat{\mathcal{T}}), Y(\mathcal{T})=Y(\hat{\mathcal{T}})$, and $f(\mathcal{T})=f(\hat{\mathcal{T}})$. (Note that $f((T, \emptyset)) \neq f((\hat{T}, \emptyset))$.) Thus $V(\mathcal{T})=V(\hat{\mathcal{T}})$ and $p(\mathcal{T})=p(\hat{\mathcal{T}})$.

In this case, in $\dot{T}$ we exchange the edge $p_{2 k-1} p_{2 k-1}^{\prime}$ for the edge $p_{2 k-2} p_{2 k}$, and the resulting triangulation is a subdivision of $T_{n-1, k-1}$. Thus $\operatorname{deg}(\dot{\mathcal{T}})=\operatorname{deg}\left(\mathcal{T}_{n-1, k-1}\right)$ and so we have

$$
\operatorname{deg}\left(\mathcal{T}_{n, k}\right) \geq \operatorname{deg}\left(\mathcal{T}_{n, k-1}\right)+\operatorname{deg}\left(\mathcal{T}_{n-1, k-1}\right) .
$$

Since we know $\operatorname{deg}\left(\mathcal{T}_{n, 0}\right)=n$, the theorem follows by solving the above recurrence.

Theorem 26 implies the following table of lower bounds, with answers known to be correct in bold.

Consider for a moment triangulations $\mathcal{T}=(T, C)$ with no subdivisions, $C=\emptyset$, and $k$ interior vertices. Example 1 is the only such triangulation with $k=1$, and of course its polynomial is linear. Up to isomorphism, Example 2 is the only such 
Table 1 Lower bounds for $\operatorname{deg}\left(\mathcal{T}_{n, k}\right)$. Values in bold are known to be sharp

\begin{tabular}{lllllllll}
\hline$n$ & 0 & 1 & 2 & 3 & 4 & 5 & 6 & 7 \\
\hline$k=0$ & $\mathbf{1}$ & $\mathbf{1}$ & $\mathbf{2}$ & $\mathbf{3}$ & $\mathbf{4}$ & $\mathbf{5}$ & $\mathbf{6}$ & $\mathbf{7}$ \\
1 & & $\mathbf{2}$ & $\mathbf{3}$ & 5 & 7 & 9 & 11 & 13 \\
2 & & & & 8 & 12 & 16 & 20 & 24 \\
3 & & & & & & 28 & 36 & 44 \\
4 & & & & & & & & 80 \\
\hline
\end{tabular}

triangulation with $k=2$, and it has degree 2 . There are exactly two isomorphism classes of these triangulations with $k=3$, namely $\mathcal{T}_{3}$ and $\mathcal{T}_{2,1}$; both have degree 3 . The triangulation $\mathcal{T}_{3,1}$ is therefore the smallest triangulation without subdivisions satisfying $\operatorname{deg}(\mathcal{T})>k$.

We close with an observation about the multiplicities of components arising in Algorithm 16. The above table contains the correct degrees if each component has multiplicity 1 , when we run the algorithm as described. However, there are other ways to run the algorithm, and for example if $k \leq \frac{n-1}{2}$ then we could instead begin with $\mathcal{T}_{n, k}$ and kill triangles $Q p_{n} p_{n-1}$ and then $S p_{n-1} p_{n}$. This would produce the recurrence

$$
\operatorname{deg}\left(\mathcal{T}_{n, k}\right) \geq \operatorname{deg}\left(\mathcal{T}_{n-1, k}\right)+1
$$

Now, if Table 1 contains the correct values for $\operatorname{deg}\left(\mathcal{T}_{n, k}\right)$ for all $n$ and $k$, then for fixed $k$ and $n \geq 2 k+1$, the linear factor arising from this factorization must occur with multiplicity $2^{k}$. In view of the discussion after Conjecture 1 , this means that the geometric intersection of $V$ with the corresponding hyperplanes consists entirely of singular points of $V$.

Acknowledgements Thanks to Dave Perkinson, Joe Buhler, Serge Tabachnikov, Phillip Andreae, Joe $\mathrm{Fu}$, and Ray Mayer for numerous stimulating conversations on this subject. Special thanks to Dave Perkinson for introducing us to this problem and for writing an early CoCoA script to compute several examples. Thanks to Phillip Andreae for producing a senior thesis [1] that included Java code to implement our algorithm before the algorithm made any sense. Finally, thanks to the Research in Pairs program at MFO (Oberwolfach) for allowing us to spend two weeks in 2011 converting ham into theorems.

\section{References}

1. Andreae, P.: Relationships between areas in a triangulation of a square. Undergraduate thesis, Emory University (2010). At http://pid.emory.edu/ark:/25593/7tpcg

2. Connelly, R.: The rigidity of suspensions. J. Differ. Geom. 13(3), 399-408 (1978)

3. Connelly, R.: Comments on generalized Heron polynomials and Robbins' conjectures. Discrete Math. 309(12), 4192-4196 (2009)

4. Connelly, R., Sabitov, I., Walz, A.: The bellows conjecture. Beitr. Algebra Geom. 38(1), 1-10 (1997)

5. Cox, D.A., Little, J., O'Shea, D.: Using Algebraic Geometry, 2nd edn. Graduate Texts in Mathematics, vol. 185. Springer, New York (2005)

6. Fedorchuk, M., Pak, I.: Rigidity and polynomial invariants of convex polytopes. Duke Math. J. 129(2), 371-404 (2005)

7. Kantor, J., Maydanskiy, M.: Triangles Gone Wild. Mass Selecta, pp. 277-288 (2003)

8. Kasimatis, E.A., Stein, S.K.: Equidissections of polygons. Discrete Math. 85(3), 281-294 (1990)

9. Manocha, D., Canny, J.F.: Algorithm for implicitizing rational parametric surfaces. Comput. Aided Geom. Des. 9(1), 25-50 (1992) 
10. Mead, D.G.: Dissection of the hypercube into simplexes. Proc. Am. Math. Soc. 76(2), 302-304 (1979)

11. Monsky, P.: On dividing a square into triangles. Am. Math. Mon. 77(2), 161-164 (1970)

12. Monsky, P.: A conjecture of Stein on plane dissections. Math. Z. 205, 583-592 (1990)

13. Pak, I.: The area of cyclic polygons: recent progress on Robbins' conjectures. Adv. Appl. Math. 34(4), 690-696 (2005)

14. Richman, F., Thomas, J.: Advanced problem 5479. Am. Math. Mon. 74(3), 329 (1967)

15. Robbins, D.P.: Areas of polygons inscribed in a circle. Discrete Comput. Geom. 12(2), 223-236 (1994)

16. Robbins, D.P.: Areas of polygons inscribed in a circle. Am. Math. Mon. 102(6), 523-530 (1995)

17. Robins, A.: A real cut-up. Undergraduate thesis, Reed College (1996)

18. Sabitov, I.Kh.: The volume as a metric invariant of polyhedra. Discrete Comput. Geom. 20(4), 405425 (1998)

19. Stein, S.: Cutting a polygon into triangles of equal areas. Math. Intell. 26(1), 17-21 (2004)

20. Stein, S.K., Szabó, S.: Algebra and Tiling: Homomorphisms in the Service of Geometry. Carus Mathematical Monographs, vol. 25. Math. Assoc. Am., Washington (1994)

21. Thomas, J.: A dissection problem. Math. Mag. 41(4), 187-190 (1968)

22. Varfolomeev, V.V.: Inscribed polygons and Heron polynomials. Mat. Sb. 194(3), 3-24 (2003) 